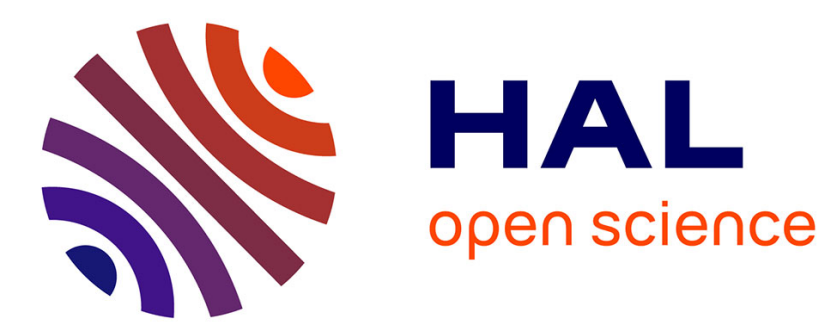

\title{
Direct numerical simulation of nucleate pool boiling at large microscopic contact angle and moderate Jakob number
}

\author{
Grégory Huber, Sébastien Tanguy, Michaël Sagan, Catherine Colin
}

\section{- To cite this version:}

Grégory Huber, Sébastien Tanguy, Michaël Sagan, Catherine Colin. Direct numerical simulation of nucleate pool boiling at large microscopic contact angle and moderate Jakob number. International Journal of Heat and Mass Transfer, 2017, vol. 113, pp. 662-682. 10.1016/j.ijheatmasstransfer.2017.05.083 . hal-01552748

\author{
HAL Id: hal-01552748 \\ https://hal.science/hal-01552748
}

Submitted on 3 Jul 2017

HAL is a multi-disciplinary open access archive for the deposit and dissemination of scientific research documents, whether they are published or not. The documents may come from teaching and research institutions in France or abroad, or from public or private research centers.
L'archive ouverte pluridisciplinaire HAL, est destinée au dépôt et à la diffusion de documents scientifiques de niveau recherche, publiés ou non, émanant des établissements d'enseignement et de recherche français ou étrangers, des laboratoires publics ou privés. 


\section{Open Archive TOULOUSE Archive Ouverte (OATAO)}

OATAO is an open access repository that collects the work of Toulouse researchers and makes it freely available over the web where possible.

This is an author-deposited version published in : http://oatao.univ-toulouse.fr/ Eprints ID : 17968

To link to this article : DOI:10.1016/j.ijheatmasstransfer.2017.05.083 URL : https://doi.org/10.1016/j.ijheatmasstransfer.2017.05.083

To cite this version : Huber, Grégory and Tanguy, Sébastien and Sagan, Michaël and Colin, Catherine Direct numerical simulation of nucleate pool boiling at large microscopic contact angle and moderate Jakob number. (2017) International Journal of Heat and Mass Transfer, vol. 113. pp. 662-682. ISSN 0017-9310

Any correspondence concerning this service should be sent to the repository administrator: staff-oatao@listes-diff.inp-toulouse.fr 


\title{
Direct numerical simulation of nucleate pool boiling at large microscopic contact angle and moderate Jakob number
}

\author{
Grégory Huber, Sébastien Tanguy*, Michael Sagan, Catherine Colin \\ IMFT, UMR 5502 CNRS-UPS-INPT, Université de Toulouse, France
}

\begin{abstract}
A B S T R A C T
In this paper, we present Direct Numerical Simulations of Nucleate Boiling on a single site in configurations involving both a large microscopic contact angle, a moderate Jakob number (less than 50) and a high density ratio between the two phases. A detailed study on the validation of the numerical simulations is presented. Several issues about the numerical modelling of the contact line are addressed in order to define a global strategy to perform accurate and predictive simulations. Benchmarks from pioneering studies (Son et al., 1999) have been reproduced with more recent numerical methods and thinner grids in order to define the most relevant strategy for successful simulations. In particular, the grid sensitivity of the solution is thoroughly investigated by performing simulations with four successive grids. The numerical results are compared favorably with experimental data, since the discrepancy between the numerical solutions and the experimental data is always less than $10 \%$ whether the departure diameter or the departure frequency are considered. The influence on the numerical solution of the thermal conduction in the solid heater is also assessed and we report that this parameter has no influence in the configurations of thick and highly conductive materials that have been considered in this study. We also present clarifications about the requirement of a specific modelling in the contact line region in order to account for a possible impact of the micro-region. Finally, based on the results of this analysis of our numerical simulations, we formulate the following unusual conclusion: the implementation of a micro-region model and an additional coupling between the overall solver and such a model is not required to perform well-resolved and accurate numerical simulations in the case of high density ratio, high microscopic contact angle (up to $30^{\circ}$ ) and moderate Jakob number. Next, we present some comparisons on the bubble shape evolution between the numerical simulations and a static force balance model, in order to investigate the mechanisms leading to the bubble detachment. Finally, we conclude this paper by presenting a parametric study, by varying the Jakob number, in order to propose a new correlation on the bubble detachment radius depending on the latter dimensionless number.
\end{abstract}

\section{Introduction}

Nucleate Boiling is a topic of interest for various industrial applications, such as heat exchangers, fluid management in micro-gravity for space applications [3] or spray cooling. Whereas some macroscopic models or semi-empirical correlations can provide realistic results in different configurations [4,53], the capabilities of such models are often limited by a lack of understanding about the microscopic details involved in the whole process. Consequently, our interest is focused on the Direct Numerical Simulation of the Nucleate Boiling of a single bubble, in order to perform low scale investigations of this physical phenomenon.

\footnotetext{
* Corresponding author.

E-mail address: tanguy@imft.fr (S. Tanguy).
}

Dhir et al. report attractive results in their pioneering work [52] on the Direct Numerical Simulations of Nucleate Boiling, however some clarifications are still required to define a well-established numerical strategy to perform predictive and well-resolved simulations. Indeed, in addition to typical algorithms for the Direct Numerical Simulation of two-phase flows (VOF, Level Set, Front Tracking), Nucleate Boiling simulations require specific numerical methods to compute the heat transfer and the phase change at the interface between the liquid and the vapor. In particular, thermodynamic conditions on the interface temperature and jump conditions on the normal velocity and on the heat flux must be imposed to ensure that the second law of thermodynamics, the mass conservation and the energy conservation will be respectively preserved across the interface. Once this step has been achieved, the boiling mass flow rate is computed from the local thermal gradients. Next, this mass flow rate must be used both 
to move the interface by taking into account the boiling velocity and to impose the jump condition on the velocity field. Many works $[7,12,21,26,32,48,51,52,58,61]$ have been dedicated to the development and the implementation of specific numerical methods to perform accurate simulations including all these features. In particular, in [58] some benchmarks have been proposed to assess the relevance of several numerical methods by comparing numerical and theoretical predictions. The following conclusions have been drawn from this study, it is showed that a smoothed Dirac function should be avoided to discretize the jump condition on the normal velocity component, because by smearing out this source term, the interface is no longer transported by the correct velocity. In the same study, the authors also shown that a significant gain in accuracy can be obtained by using quadratic extrapolations of the temperature field to populate ghost cells in each side of the interface before computing the boiling mass flow rate. However in the computations presented in the latter study, no contact line was involved between a liquid-vapor interface and a solid. If one considers the Direct Numerical Simulation of Nucleate Boiling, additional issues must be faced, such as the computation of mechanical and thermal effects on the triple line which is formed where the bubble is contacting the hot wall. Modelling heat and mass transfer in the vicinity of the contact line is challenging because of the strong localization and singular behavior of the wall shear stress, the heat flux, and thus the boiling mass flow rate. For instance, the interface temperature is equal to the saturation temperature [16], corresponding to the external pressure, and can be different from the wall temperature. This temperature discontinuity on the contact line leads to a thermal singularity since the corresponding thermal flux locally tends to infinity. In the following works $[19,35,36,40,44,54,60]$, models have been proposed to develop a singularity-free description of hydrodynamics and heat transfer in the liquid micro region, which can appear in the contact line vicinity. The high evaporation rate at the contact line induces a strong pressure gradient in the micro region and a strong change in the interface curvature. At the contact line, the microscopic contact angle $\theta_{\text {mic }}$ is equal to the Young-Laplace angle and the slope of the interface increases in the micro-region, and at macroscopic scale it reaches an apparent contact angle value $\theta_{\text {app }}$. The contact line models provide information on the apparent contact angle and heat flux or mass flux in the micro-region depending on the wall superheat and on the microscopic contact angle.

We present in this paper a discussion and some results on a micro-region model proposed in former studies $[35,36]$. By using this micro region model, it will be showed that in the considered configuration, i.e. a partially wetting fluid with a high microscopic contact angle (up to $30^{\circ}$ ) and a moderate Jakob number (less than 50 ), the apparent contact angle is almost equal to the microscopic contact angle, and the mass flux is negligible by comparison to the overall mass flux around the bubble.

Next, we present new results on the Direct Numerical Simulation of Nucleate Boiling in order to bring out an efficient numerical methodology to succeed these simulations. Specific investigations have been carried out on the influence of the grid size and on the local variation of the temperature field inside the hot substrate. Once the spatial convergence of the computation has been showed, accurate comparisons with experimental data provided in [52] are presented. The numerical results are in good agreement, with the experiments, better than for works previously published, since the discrepancy between the numerical simulations and the experimental data are close to $5 \%$ both for the detachment radius and the detachment frequency of the bubble. In anterior papers, errors close to $20 \%$ are frequently reported, such as in $[26,52]$, whether the detachment radius or the detachment frequency are considered. The analysis of the difference between numerical simulations and experimental data enables discussing about the relevance of the overall model. Thus by linking the information from the Direct Numerical Simulation and from the micro-region model, we conclude our study by remarking that in the situation considered herein, a coupling between a micro-region model with the overall numerical solver is not required.

Next we propose a study of the bubble shape during its growth. The bubble shape obtained with the Direct Numerical Simulation is compared to the static bubble shape calculated from the integration of the Laplace equation. These comparisons show a good agreement in the initial step of the bubble growth when the contact line is advancing in the liquid domain, whereas some differences appear during the receding phase. This difference can be attributed to the mass added force, induced by the liquid inertia, which cannot be neglected when the contact line is receding. Indeed, when the contact line is receding, the interface motion is no longer driven by slow thermal effect but it is accelerated by the development of a capillary instability. Finally, a parametric study is presented to determine the influence of the Jakob number on the bubble radius detachment.

\section{Physical model and numerical methods}

\subsection{Conservation laws and jump conditions}

The Direct Numerical Simulation of Nucleate Boiling is performed by solving the conservation laws resulting from the primitive principles of mass conservation, momentum balance, energy conservation and the second law of thermodynamics. As we will consider moderate wall superheat and low Mach number flows, we will assume that the densities of the fluids are spatially uniform. Moreover, all the simulations presented in this paper will contain a free boundary condition in order to maintain isobaric conditions in the computational field. Thus, we can assume that the free-divergence condition will be respected in each phase

$\nabla \cdot \vec{V}=0$

where $\vec{V}$ is the velocity field.

However, following the jump condition formulation [50], if phase change occurs, the following jump condition on the velocity field must be imposed to ensure the mass conservation at the interface $[12,38,57]$

$[\vec{V}]_{\Gamma}=\dot{m}\left[\frac{1}{\rho}\right]_{\Gamma} \vec{n}$

where $\dot{m}$ is the local vapor mass flow rate, $\rho$ is the fluid density, $\vec{n}$ is the normal vector at the interface pointing in the direction of the liquid phase. The operator $[\cdot]_{\Gamma}$ denotes a jump condition across the interface and it is defined as follows

$[f]_{\Gamma}=f_{\text {vap }}-f_{\text {liq }}$.

The momentum balance can be expressed with the NavierStokes equations for an incompressible flow

$\rho \frac{D \vec{V}}{D t}=-\nabla p+\nabla \cdot(2 \mu \boldsymbol{D})+\rho \vec{g}$,

where $p$ denotes the pressure, $\mu$ the viscosity, $\boldsymbol{D}$ the deformation tensor and $\vec{g}$ the acceleration vector due to gravity. In order to impose the momentum balance at the interface, an additional jump condition on pressure must be satisfied across the interface

$[p]_{\Gamma}=\sigma \kappa+2\left[\mu \frac{\partial V_{n}}{\partial n}\right]_{\Gamma}-\dot{m}^{2}\left[\frac{1}{\rho}\right]_{\Gamma}$ 
where $\sigma$ is the surface tension, $\kappa$ is the local curvature of the interface and $\frac{\partial V_{n}}{\partial n}$ is the derivative in the normal direction of the normal velocity component at the interface. The first term of the right hand side of this equation is the capillary pressure from the Laplace-Young law, the second term accounts for the discontinuity of the normal viscous stress, and the last term is usually referred as the recoil pressure. As for single component liquid-vapor systems, the interface temperature is uniform and equal to the saturation temperature, a constant value of the surface tension is imposed. As a result the jump of the tangential viscous stresses across the interface is equal to zero. It is noteworthy to remind that weak variations of the saturation temperature can occur locally due to the pressure jump condition, such as it is the case for the Kelvin effect which is induced by the variation of the saturation temperature due to a non-uniform capillary pressure. In specific situations these variations can imply a significant discontinuity of the tangential viscous stresses and leads to the development of a Marangoni convection even if single component liquid-vapor systems are considered. In the Ref. [21], the authors proposed a quantitative study on the respective scale of the surface tension variations for different phenomena, in order to bring out that corrections are negligible in most configurations.

Finally, if we assume that the heating due to viscous dissipation can be neglected and that our system is isobaric, the first law of thermodynamics can be expressed with the following simplified equation based on an enthalpy formulation

$\rho C_{p} \frac{D T}{D t}=\nabla \cdot(k \nabla T)$.

$T$ is the temperature, $C_{p}$ is the specific heat for a constant pressure, and $k$ is the thermal conductivity. An additional jump condition on the thermal flux is imposed to account for the latent heat of vaporization

$[-k \nabla T \cdot \vec{n}]_{\Gamma}=\dot{m}\left(L_{v a p}-\left[C_{p}\right]_{\Gamma}\left(T_{s a t}-\left.T\right|_{\Gamma}\right)\right)$.

$L_{v a p}$ is the latent heat of vaporization, $T_{\text {sat }}$ is the saturation temperature of the liquid depending on the external pressure and $\left.T\right|_{\Gamma}$ is the interface temperature. By considering that the interface temperature is equal to the saturation temperature for a single component liquid and vapor phase, the latter jump condition can be simplified

$[-k \nabla T \cdot \vec{n}]_{\Gamma}=\dot{m} L_{\text {vap }}$

As it will be further detailed, this jump condition will be used in the simulations to compute the vapor mass flow rate.

\subsection{Numerical methods}

The numerical methods used in this study are based on the pioneering work of Fedkiw et al [8] on the Ghost Fluid Method. This preliminary work has instigated many other numerical methods which have been developed keeping the guidelines of this first paper $[11-13,15,22,29,33,34,38,39,43,45,56-58]$. All these studies present new developments of numerical methods allowing a sharp description of discontinuous terms in the framework of a welldefined numerical theory. We summarize here briefly the numerical methods developed in this paper to perform accurate direct numerical simulations of two-phase flows with phase change. The Level Set Method [42,55] is used to compute the interface motion by solving a convection equation for a Level Set Function $\phi$

$\frac{\partial \phi}{\partial t}+\vec{V}_{i n t} \cdot \nabla \phi=0$

Positive values of $\phi$ represent the liquid field and the negative values in the gas field. $\vec{V}_{i n t}$ is the interface velocity
$\vec{V}_{\text {int }}=\vec{V}_{\text {liq }}+\frac{\dot{m}}{\rho_{\text {liq }}} \vec{n}=\vec{V}_{\text {vap }}+\frac{\dot{m}}{\rho_{\text {vap }}} \vec{n}$

Next, as proposed in [55], a reinitialization step is carried out in order to ensure that the $\phi$ function remains a signed distance in the computational field. This is done by solving iteratively the following algorithm

$\frac{\partial d}{\partial \tau}=\operatorname{sign}(\phi)(1-|\nabla d|)$

with $d$ the reinitialized distance function, $\tau$ a fictitious time and sign $(\phi)$ is a smoothed signed function defined in [55]. Two temporal iterations of the resistance equation, Eq. (11), are performed at every time step, in order to preserve a sufficient accuracy for the computation of the normal vector (pointing to the liquid domain) and of the curvature at the interface

$\vec{n}=\frac{\nabla \phi}{\|\nabla \phi\|}$

$\kappa(\phi)=-\nabla \cdot \vec{n}$

Spatial derivatives are computed with usual numerical schemes, such as fifth order WENO (Weighted Essentially Non Oscillatory) scheme [20] for the convective derivatives and second order finite volume for the diffusion terms. Temporal integration is carried out with a second order Runge Kutta scheme. In recent works $[28,30]$ accurate comparisons between experimental data and Direct Numerical Simulations on oscillations of bubble rising have been presented by using these numerical methods. It is noteworthy that in the following papers $[5,37,47]$, an alternative method is proposed to compute the distance function by using a subcell fix in the grid cells that are crossed by the interface. This technique enables not using a smoothed sign function during the reinitialization step.

As the interface temperature is constant and continuous across the interface, the following algorithm, named GFTSB (Ghost Fluid Thermal Solver for Boiling) in [45], has been designed in [12] to solve the heat transfer around the interface when phase change occurs. First, solve the temperature field in the liquid domain with a prescribed Dirichlet boundary condition at the interface

$\rho_{l} C p_{l} T_{l}^{n+1}-\Delta t \nabla \cdot\left(k_{l} \nabla T_{l}^{n+1}\right)=\rho_{l} C p_{l}\left(T_{l}^{n}-\Delta t \vec{V}_{l}^{n} \cdot \nabla T_{l}^{n}\right)$

if $\phi>0,\left.T\right|_{\Gamma}=T_{\text {sat }}$,

with $\phi$ a Level Set function. Next, solve the temperature field in the vapor domain with the same prescribed Dirichlet boundary condition at the interface

$\rho_{v} C p_{v} T_{v}^{n+1}-\Delta t \nabla \cdot\left(k_{v} \nabla T_{v}\right)=\rho_{v} C p_{v}\left(T_{v}^{n}-\Delta t \vec{V}_{v}^{n} \cdot \nabla T_{v}^{n}\right)$

if $\phi<0,\left.\quad T\right|_{\Gamma}=T_{\text {sat }}$.

Imposing an immersed Dirichlet boundary condition on an interface, described on a Cartesian grid, can be done by using the numerical method proposed by Gibou et al. in [11]. This efficient method is second order accurate in space and leads to a simple symmetric definite positive linear system that can be solved with a standard Black Box solver. It should be mentioned that second order and sharp discretization have also been proposed for immersed Neumann and Robin boundary condition, respectively in the following Refs. $[39,43]$. Once the temperature field has been computed, the local mass flow rate can be easily deduced from

$\dot{m}=\frac{[-k \nabla T \cdot \vec{n}]_{\Gamma}}{L_{\text {vap }}}$ 
In order to compute accurately the thermal gradients across the interface, extrapolations techniques are required to extend continuously the temperature field and its derivatives by populating ghost cells in each side of the interface [1]. This is done by solving three successive Partial Differential Equations to extend the vapor temperature field in the liquid domain. Theses equations are

$\frac{\partial T_{n n}}{\partial \tau}+H(\phi) \vec{n} \cdot \nabla T_{n n}=0$,

$\frac{\partial T_{n}}{\partial \tau}+H(\phi)\left(\vec{n} \cdot \nabla T_{n}-T_{n n}\right)=0$

$\frac{\partial T}{\partial \tau}+H(\phi)\left(\vec{n} \cdot \nabla T-T_{n}\right)=0$

where $\tau$ is a fictitious time, $H(\phi)$ is an Heaviside function corresponding to the Level Set function $\phi, T_{n}$ and $T_{n n}$ are respectively the first and second derivative in the normal direction. Theses equations are solved as an iterative process until a steady state is reached. A similar set of equations must also be solved to extend the liquid temperature field in the vapor domain

$\frac{\partial T_{n n}}{\partial \tau}-H(-\phi) \vec{n} \cdot \nabla T_{n n}=0$,

$\frac{\partial T_{n}}{\partial \tau}-H(-\phi)\left(\vec{n} \cdot \nabla T_{n}-T_{n n}\right)=0$,

$\frac{\partial T}{\partial \tau}-H(-\phi)\left(\vec{n} \cdot \nabla T-T_{n}\right)=0$.

More details on the implementation of these extensions are provided in [1]. Let us notice, that in a recent paper [58], it has been showed that extending the temperature, the first normal derivative and the second normal derivative strongly improves the overall accuracy of the computation.

Once $\dot{m}$ has been determined, the jump condition on the normal velocity can be imposed when solving Navier-Stokes equations. We describe here briefly the numerical method which has been proposed in [38] in the framework of premixed front flame propagation. Next, this method has been used to deal with boiling flows, such as in [12,58], and improved to deal with droplet in $[45,46,57]$. We use in this paper an implicit temporal discretization of the viscous terms that have been proposed in [29] and referred in the latter as the implicit Ghost Fluid Semi-Conservative viscous Method. This numerical scheme has already been used by Rueda Villegas et al. $[45,46]$ to perform numerical simulations of droplet levitating above a hot plate in the Leidenfrost regime. In this specific regime, a very refined grid (with a minimum length of the grid cell which is approximately $1 \mu \mathrm{m}$ ) must be used in the vicinity of the wall to capture the formation of a very thin vapor layer between the bottom of the droplet and the hot plate. Thus, using an explicit temporal discretization leads to drastic constraints on the time step, especially for the constraints from diffusion terms which vary as the square of the smallest length of the grid cell. Therefore, an implicit temporal discretization of these diffusion terms, due to the viscosity and the thermal conduction, is essential to alleviate the time step constraint in order to speed-up the simulations. In the case of Nucleate Boiling simulations, the length of the grid cell is not as small as for simulations of Leidenfrost droplet. However for the purpose of the convergence study which will be presented in the results section, a very well resolved simulation with a grid cell length of $5 \mu \mathrm{m}$ is presented. In the latter configurations, the time step due to viscosity is more drastic than the time step due to capillary effect, and an implicit temporal discretization of the viscous terms is beneficial considering the computational cost.
We remind here the implicit temporal discretization of the viscous terms that has been used in $[29,45]$ when the Ghost Fluid Semi-Conservative viscous Method is used with a projection method for solving incompressible flows

$\rho^{n+1} \vec{V}^{*}-\Delta t \nabla \cdot\left(\mu \nabla \vec{V}^{*}\right)=\rho^{n+1}\left(\vec{V}^{n}-\Delta t\left(\vec{A}\left(\vec{V}^{n}\right)-\vec{g}\right)\right)$,

with the following definition of $\vec{A}\left(\vec{V}^{n}\right)$ to account for convective terms if phase change occurs

$$
\begin{aligned}
& A^{\rightarrow}\left(V^{\rightarrow n}\right)=V_{l}^{\rightarrow n} \cdot \nabla V_{l}^{\rightarrow n} \text { if } \phi>0, \\
& A^{\rightarrow}\left(V^{\rightarrow n}\right)=V_{g}^{\rightarrow n} \cdot \nabla V_{g}^{\rightarrow n} \text { if } \phi<0 .
\end{aligned}
$$

The projection of Eq. (24) on the x-axis and the y-axis leads respectively to the following relation on a staggered grid

$$
\begin{aligned}
& \rho_{i+1 / 2 j}^{n+1} u_{i+1 / 2 j}^{*}-\left.\Delta t\left(\nabla \cdot\left(\mu \nabla u^{*}\right)\right)\right|_{i+\frac{1}{2} j} \\
& \quad=\rho_{i+\frac{1}{2} j}^{n+1}\left(u_{i+\frac{1}{2} j}^{n}-\Delta t\left(\left.\left(\vec{A}\left(\vec{V}^{n}\right) \cdot \vec{e}_{x}\right)\right|_{i+\frac{1}{2} j}-\vec{g}\right)\right), \\
& \rho_{i j+1 / 2}^{n+1} v_{i j+1 / 2}^{*}-\left.\Delta t\left(\nabla \cdot\left(\mu \nabla v^{*}\right)\right)\right|_{i j+1 / 2} \\
& =\rho_{i j+1 / 2}^{n+1}\left(v_{i j+1 / 2}^{n}-\Delta t\left(\left.\left(\vec{A}\left(\vec{V}^{n}\right) \cdot \vec{e}_{y}\right)\right|_{i j+1 / 2}-\vec{g}\right)\right) .
\end{aligned}
$$

This temporal discretization allows computing separately each component of the velocity field by solving a symmetric positive definite linear system. If phase change occurs, the following two scalar jump conditions must be imposed when solving the previous two equations to preserve the mass conservation across the interface

$\left[u_{i+1 / 2 j}^{*}\right]=\dot{m}_{i+1 / 2 j}\left[\frac{1}{\rho}\right]_{\Gamma} n_{i+1 / 2 j}^{x}$,

$\left[v_{i j+1 / 2}^{*}\right]=\dot{m}_{i j+1 / 2}\left[\frac{1}{\rho}\right]_{\Gamma} n_{i j+1 / 2}^{y}$.

The spatial discretization proposed in [34] is used to impose these jump conditions when solving Eqs. (26) and (27). Next, the velocity field must be extended in each side of the interface to populate ghost cells, with the following relations

$$
\begin{aligned}
& \begin{cases}V_{\text {liq }}^{\rightarrow *}=V^{\rightarrow *} & \text { if } \phi>0, \\
V_{\text {liq }}^{\rightarrow \text { ghost }}=V^{\rightarrow *}+\dot{m}\left[\frac{1}{\rho}\right]_{\Gamma} n^{\rightarrow} & \text { if } \phi<0\end{cases} \\
& \begin{cases}V_{\text {vap }}^{\rightarrow *}=V^{\rightarrow *} & \text { if } \phi<0, \\
V_{\text {vap }}^{\rightarrow \text { ghost }}=V^{\rightarrow *}-\dot{m}\left[\frac{1}{\rho}\right]_{\Gamma} n^{\rightarrow} & \text { if } \phi>0 .\end{cases}
\end{aligned}
$$

In the following step, the Poisson equation for the pressure is computed

$\nabla \cdot\left(\frac{\nabla p^{n+1}}{\rho^{n+1}}\right)=f$

where the velocity extension is used to compute the right hand side $f$, as follows

$$
\begin{cases}f=\frac{\nabla \cdot \vec{V}_{\text {liq }}^{*}}{\Delta t} & \text { if } \phi>0 \\ f=\frac{\nabla \cdot \vec{V}_{\text {vap }}^{*}}{\Delta t} & \text { if } \phi<0,\end{cases}
$$

and with the pressure jump condition which is imposed with the numerical methods proposed in $[22,34]$

$[p]_{\Gamma}=\sigma \kappa+\left[\mu \frac{\partial V_{n}}{\partial n}\right]_{\Gamma}-\dot{m}^{2}\left[\frac{1}{\rho}\right]_{\Gamma}$. 
The last step is the correction of the velocity field to ensure that the velocity field is free-divergence in the two phases (but not across the interface according to the jump condition given by Eq. (2)). This correction is expressed as follows if the GFSCM is used in conjunction with phase change:

$\vec{V}^{n+1}=\vec{V}^{*}-\frac{\Delta t}{\rho^{n+1}}\left(\nabla P^{n+1}-\left(\sigma \kappa+\left[\mu \frac{\partial V_{n}}{\partial n}\right]_{\Gamma}-\dot{m}^{2}\left[\frac{1}{\rho}\right]_{\Gamma}\right) \vec{n} \delta_{\Gamma}\right)$.

More details on the implementation of these different steps can be found in the following Refs. [29,34,45].

In [57], an additional step is performed to compute a ghost field for the pressure in order to build an extension of the velocity field which respects the divergence-free condition at the interface. This further step strongly improves the mass conservation when considering droplet vaporization. However, it has been shown in [58], that its impact was not so crucial in the framework of boiling flows when bubble growth is involved. Consequently, as this correction is computationally expensive (one more Poisson equation must be solved), it is not used in this study. Classical time step constraints must be imposed to ensure the temporal stability in regards with convective terms, and surface tension terms

$\Delta t_{\text {conv }}=\frac{\Delta x}{\max \|\vec{V}\|}$,

$\Delta t_{\text {surf_tens }}=\frac{1}{2} \sqrt{\frac{\rho_{\text {liq }} \Delta x^{3}}{\sigma}}$.

The global time step restriction which accounts for the previous two constraints is given by

$\frac{1}{\Delta t}=\frac{1}{\Delta t_{\text {conv }}}+\frac{1}{\Delta t_{\text {surf_tens }}}$

The central diagonal of the linear systems resulting from Eqs. (26) and (27) are very dominant due to the low time steps (which are mainly limited by the capillary constraint) used in all simulations. Therefore, a fast resolution of these linear systems can be carried out by using an Incomplete Choleski Conjugate Gradient method (ICCG).

Finally, in the framework of the direct numerical simulation of Nucleate Boiling, a prescribed contact angle $\theta$ must be imposed on the wall. That can be done by imposing the following Neumann boundary condition on the $\phi$ function, such as it is proposed in [33]

$\left.\vec{n} \cdot \nabla \phi\right|_{=}-\cos \theta$.

As the two equations for the $\phi$ function, Eqs. (9) and (11), are solved with an explicit temporal discretization, this boundary condition can be imposed by populating ghost cells outside of the computational field. If an immersed solid domain is considered inside the computational field, solving an iterative PDE is required, such as in $[1,8,33]$, to build an extrapolated field that maintains the suitable boundary condition on the $\phi$ function for imposing the contact angle. In the simulations presented in this paper, as a horizontal wall in the x-direction with a normal vector in the $\mathrm{y}$ direction is considered, the following simple relation allows imposing this boundary condition:

$\phi_{i, j=0}=\phi_{i, j=1}+\Delta y \cos \theta$.

\section{About micro region model for nucleate boiling of partially wetting fluids}

We present in this section a discussion about the relevance of a coupling between the overall solver and a heat flux subgrid model accounting for a micro-region that could be formed in the vicinity of the contact line. Some experimental evidences of such a microregion have already been reported in the literature, for instance by indirect measurements on the wall heat flux. In particular, this often results in a strong increase of the heat flux due to the large thermal gradient in the micro-region. The interested reader will find more details on this topic in the following Refs. $[18,19,27,44,54,60]$.

At macroscopic scale, the observed contact angle between the liquid-vapor interface and the wall is named the apparent contact angle or macroscopic contact angle $\theta_{a p p}$. As illustrated in Fig. 1, at microscopic scale, a strong evolution of the contact angle can be observed from $\theta_{\text {mic }}$ to $\theta_{\text {app }}$. This microscopic contact angle $\theta_{\text {mic }}$, results from a mechanical equilibrium of the triple line between the three phases and its value is given by Young's Equation. In configurations without any phase change, where a static bubble or droplet is considered, there is no difference between the microscopic contact angle and the apparent contact angle. On the other hand, if Nucleate Boiling is considered, the apparent contact angle can differ from the microscopic angle in a way that depends on the wall superheat, $T_{\text {wall }}-T_{\text {sat }}$, and on the microscopic contact angle. The liquid region under the interface before it forms the apparent contact angle is named the micro-region.

As stated in a recent work by Fischer et al [9], this micro-region whose size is typically less than $1 \mu \mathrm{m}$, should not be confused with the microlayer. Indeed, the micro-region is formed due to the influence of attractive intermolecular forces between molecules of the solid phase and molecules of the fluid phases. These forces locally modify the interface profile, and thus the heat flux in the vicinity of the contact line. On the other hand, a microlayer can be formed at higher Jakob number (in fact other parameters as Reynolds number and Prandtl number must be considered to characterize the frontier between the two regimes) when the interface velocity is so fast, that the contact line cannot advance at the same speed because it is slowed down by the wall viscous friction. This microlayer whose formation is essentially driven by hydrodynamics effects, as described by Landau and Levich in [31], can be much thicker (several tens of $\mu \mathrm{m}$ following [9]) than the micro-region which is formed in the vicinity of the contact line at lower Jakob number. Also, it can be much longer since the same authors report some experimental evidences of $2 \mathrm{~mm}$ long evaporating films, such as it can be observed in Fig. 5 in Ref. [9]. This microlayer was also observed in [25] in boiling experiments by Kim and Buongiorno.

The numerical modelling of these two distinct phenomena, occurring at different scales, will imply different numerical strategies. On one hand computing the flow, the heat and mass transfer into the micro-region with the same solver as for the overall bubble computation is currently not conceivable. Indeed, the latter task would require too small cells to capture the micro-region. For instance, in the simulations presented in this paper, the smallest cell length used is $5 \mu \mathrm{m}$ whereas the micro region size can be typically under $1 \mu \mathrm{m}$. For this reason, a specific model must be developed to account for this micro region. As stated previously, two kinds of micro-region model exist: perfectly wetting fluid micro-region model as in [54] and partially wetting fluid microregion models which have been developed in $[19,35,36,40]$. On the other hand performing a fully resolved Direct Numerical Simulation of Nucleate Boiling when a microlayer will develop seems to be a more feasible task, since the range of scales involved in the overall physical phenomenon is not as large as in the case involving a micro-region. Recent studies have presented first results on such a configuration by using a very refined grid in the vicinity of the wall in [14], or by using a subgrid model to describe this micro-layer in [49].

Although numerical simulations of Nucleate Boiling have already been presented in many different works [26,48,52], there 


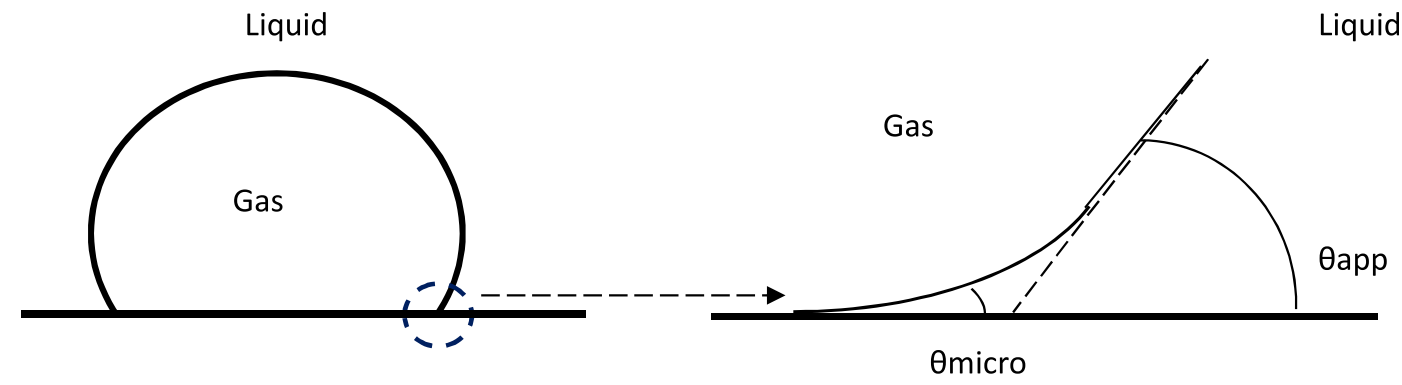

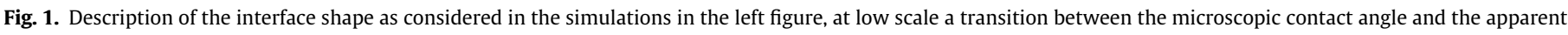
contact angle can be observed as in the right figure.

is currently no consensus in the community on the way to account for the micro-region when performing Direct Numerical Simulations of Nucleate Boiling. Indeed, the development of such models is still an active field of research, and, depending on the considered configurations, the influence on the overall bubble growth of this micro-region can be versatile [24]. In particular, configurations involving perfectly wetting fluids or partially wetting fluids must be distinguished, since important differences on the contact line behavior can be observed following which one of the two options is considered.

Based on the benchmarks previously proposed by Son et Dhir in [52], we will present, in this paper, computations involving Nucleate Boiling of water vapor on a hot plate (silicon wafer), i.e.; a partially wetting fluid with a large contact angle (up to $30^{\circ}$ ). For the purpose of this study, the micro-region model proposed by Mathieu et al. [35,36], has been implemented in order to quantify the influence of the wall superheat on the apparent contact angle in configurations for which the microscopic contact angle is large. Moreover, this preliminary study will enable us to evaluate the magnitude of the vaporization flux in the micro-region before considering a coupling of this micro-region model with the overall solver.

We present here briefly the micro-region model developed by Mathieu et al. in $[35,36]$. This model can be understood as a generalization to partially wetting fluid of the model for perfectly wetting fluid presented by Stephan and Büsse in [54]. It is composed to the following set of five equations

$$
\begin{aligned}
& \frac{\partial x}{\partial s}=\cos \theta \\
& \frac{\partial y}{\partial s}=\sin \theta \\
& \frac{\partial \theta}{\partial s}=\frac{\Delta p}{\sigma}
\end{aligned}
$$

$\frac{\partial(\Delta p)}{\partial s}=-\mu_{l} \frac{3 Q}{\rho_{l} L_{v a p} \theta^{3}}$,

$\frac{\partial Q}{\partial s}=k_{l} \frac{\Delta T}{r \theta+k R_{i}}$,

where $s$ is the curvilinear abscissa, $\theta(s)$ is the angle between the interface and the wall, $\Delta P(s)$ is the pressure jump through the interface, $Q(s)$ is the integrated energy flux from the triple line to $s, R_{i}$ is the interface resistance, $\sigma$ is the surface tension, $\mu_{1}$ is the liquid dynamic viscosity, and $k_{l}$ is the liquid thermal conductivity. As partially wetting fluids are considered, the model does not assume the formation of an adsorbed liquid film between the vapor and the wall. Consequently the disjoining pressure can be neglected since it has no significant effect at large scale, such as it has been reported in [19], when partially wetting liquids are considered; i.e. if there is no adsorbed liquid film. However a slip length $l_{s}$ is introduced in Eq. (45) to obtain a singularity free model considering the hydrodynamic. $\Delta T$ is defined as the wall superheat $\Delta T=T_{\text {wall }}-T_{\text {sat }} . T_{\text {sat }}$ is modified due to the Kelvin effect which accounts for the variation of the saturation temperature with the pressure jump due to capillary effects and it can be expressed as $T_{\text {sat }}=T_{\text {sat }, 0}\left(1+\frac{\Delta p}{\rho_{L} L_{\text {vap }}}\right)$, where $L_{v a p}$ is the latent heat of vaporization. Finally $r$ is defined as $\frac{y+l_{s}}{\sin \theta}, y$ being the distance from the horizontal wall and the interfacial resistance can be expressed as $R_{i}=\frac{(2-\varepsilon) T_{s a t} \sqrt{2 \pi R_{g} T_{\text {sat }}}\left(\rho_{l}-\rho_{v}\right)}{2 \varepsilon \rho_{v} \rho_{l} L_{\text {vap }}^{2}}$, where $\varepsilon$ is the accommodation coefficient taken equal to 1 and $R_{g}=R / M$, $R=8.314 \mathrm{~J} \mathrm{~mol}^{-1} \mathrm{~K}^{-1}$ being the universal gas constant and $M$ the molar mass of the fluid.

The first two Eqs. (41) and (42) connect the geometrical coordinates of each point on the interface. The third equation describes the angle evolution due to curvature. The fourth equation derives from the Stokes equation and accounts for the pressure drop along the interface. The recoil pressure does not appear in this equation. Indeed, it can be neglected when moderate Jakob numbers are involved, as it is the case in this study. The fifth equation is the expression of the conduction flux from the wall to the interface, considering the interface thermal resistance and the Kelvin effect. For solving this system of equations, a numerical algorithm, based on a shooting method and a Runge Kutta scheme to compute the derivatives, is briefly described in the Appendix A. A steady-state can be assumed when solving the governing equations in the micro-region. Indeed, Mathieu's model being applied at very low scales in the vicinity of the interface, viscosity and conduction are the dominant effects in comparison with inertia and convection. Moreover, low contact line velocities (about $0.01 \mathrm{~m} \mathrm{~s}^{-1}$ ) are involved in our simulations, thus there is no significant effect of the contact line velocity on the apparent contact angle, such as it can be observed for high speed droplet impact.

\section{Numerical results}

At first, we present results on the contact line model, described in the previous section, with several physical conditions. In particular, we demonstrate that in the specific, but usual, case involving a moderate Jakob number, a large microscopic contact angle and a high density ratio, the apparent contact is almost equal to the microscopic contact angle, and the micro-region heat flux can be neglected. From these preliminary results, we will assume that a coupling between the overall solver and a micro-region model is not required in the vicinity of the contact line in configurations 
Table 1

Physical properties of the liquid water and of the steam water for an ambient pressure and $T=373 \mathrm{~K}$.

\begin{tabular}{|c|c|c|c|c|c|c|}
\hline Physical properties (SI) & $\rho$ & $M$ & $k$ & $C p$ & $\Sigma$ & $L v$ \\
\hline Liquid & 958 & $2.82 \mathrm{E}-4$ & 0.677 & 4216 & 0.058 & $2.256 \mathrm{E} 6$ \\
\hline Vapor & 0.5974 & $1.228 \mathrm{E}-5$ & 0.024 & 2034 & - & - \\
\hline
\end{tabular}

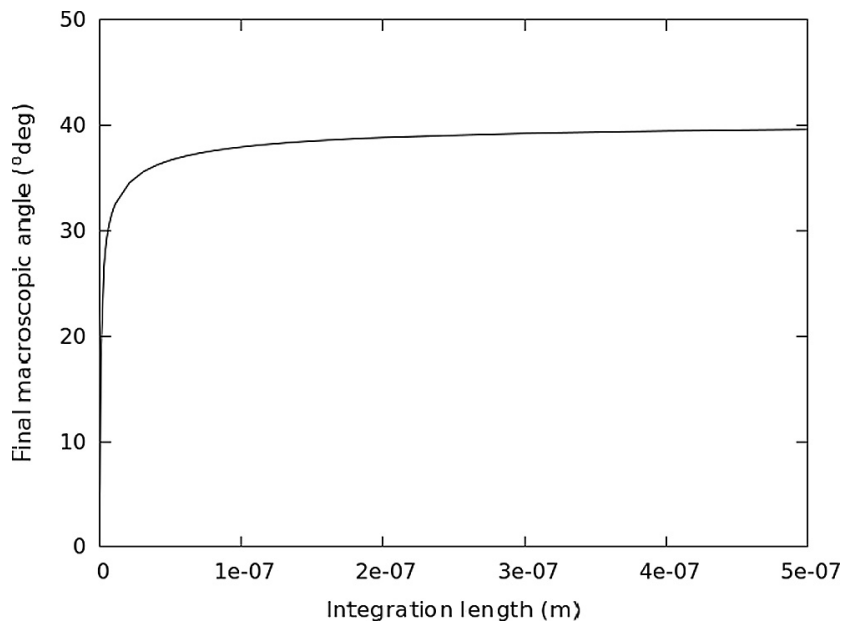

Fig. 2. Final macroscopic contact angle as a function of the total integration length with the following parameters $\Delta T=100 \mathrm{~K}, \theta_{\text {mic }}=5^{\circ}$.
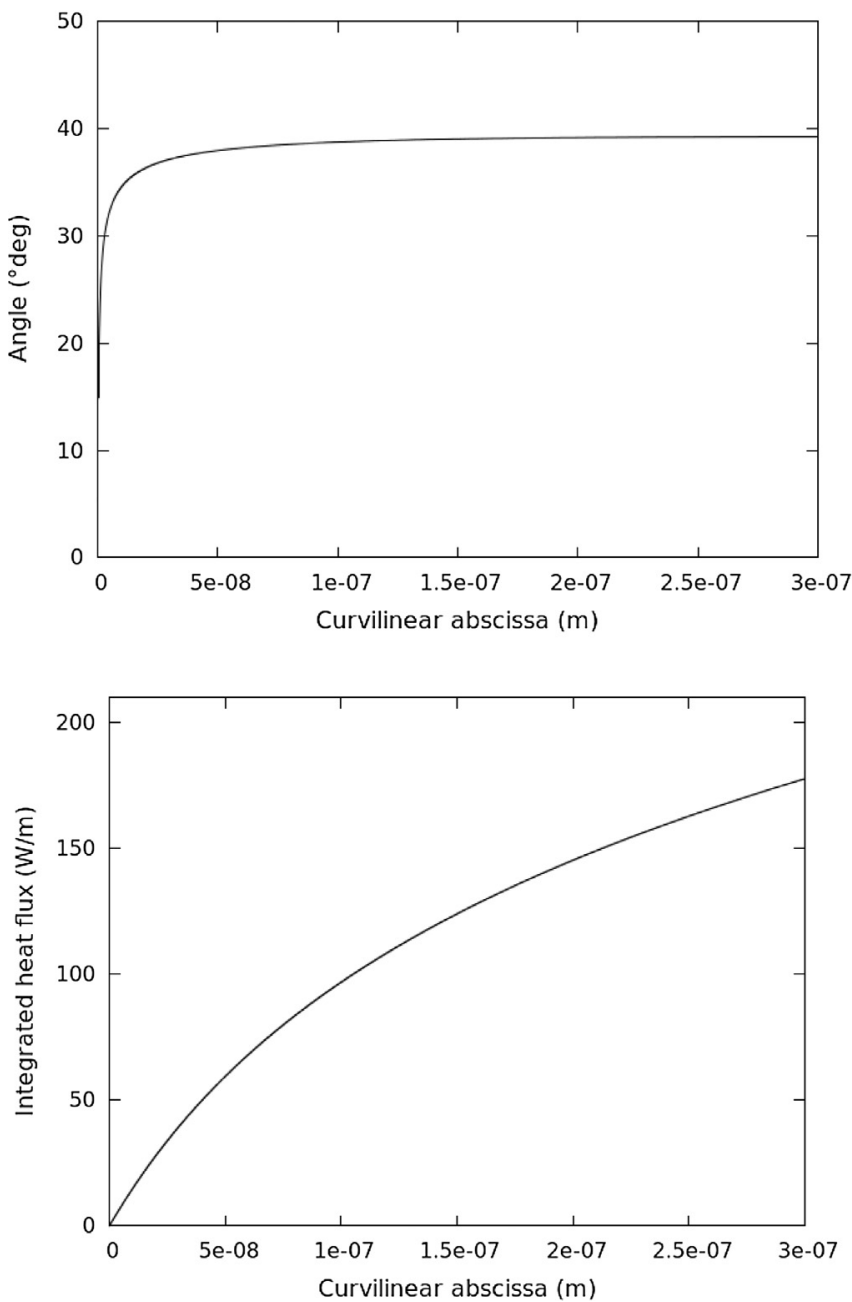

implying a large microscopic contact angle, a moderate Jakob number and a high density ratio between the two phases (such as it is the case for most ambient pressure applications). Configurations involving lower microscopic contact angle (less than $30^{\circ}$ ) would require a more detailed description of the micro-region involving a local subgrid heat flux in the grid cells crossed by the contact line. When considering nucleate boiling with higher Jakob number, a hydrodynamic microlayer can expand and lead to a very different behavior of the bubble growth and a larger heat transfer coefficient. To the best of our knowledge, the critical Jakob number, for which the transition between the contact line regime and the microlayer regime occurs, is not known currently. It is expected that this critical value depends strongly of the apparent contact angle.

Next, we present, Direct Numerical Simulations of Nucleate Boiling without any micro-region model, in configurations where the previous conditions are respected. These simulations are compared to the experimental data by Son et al. in [52] with water. The
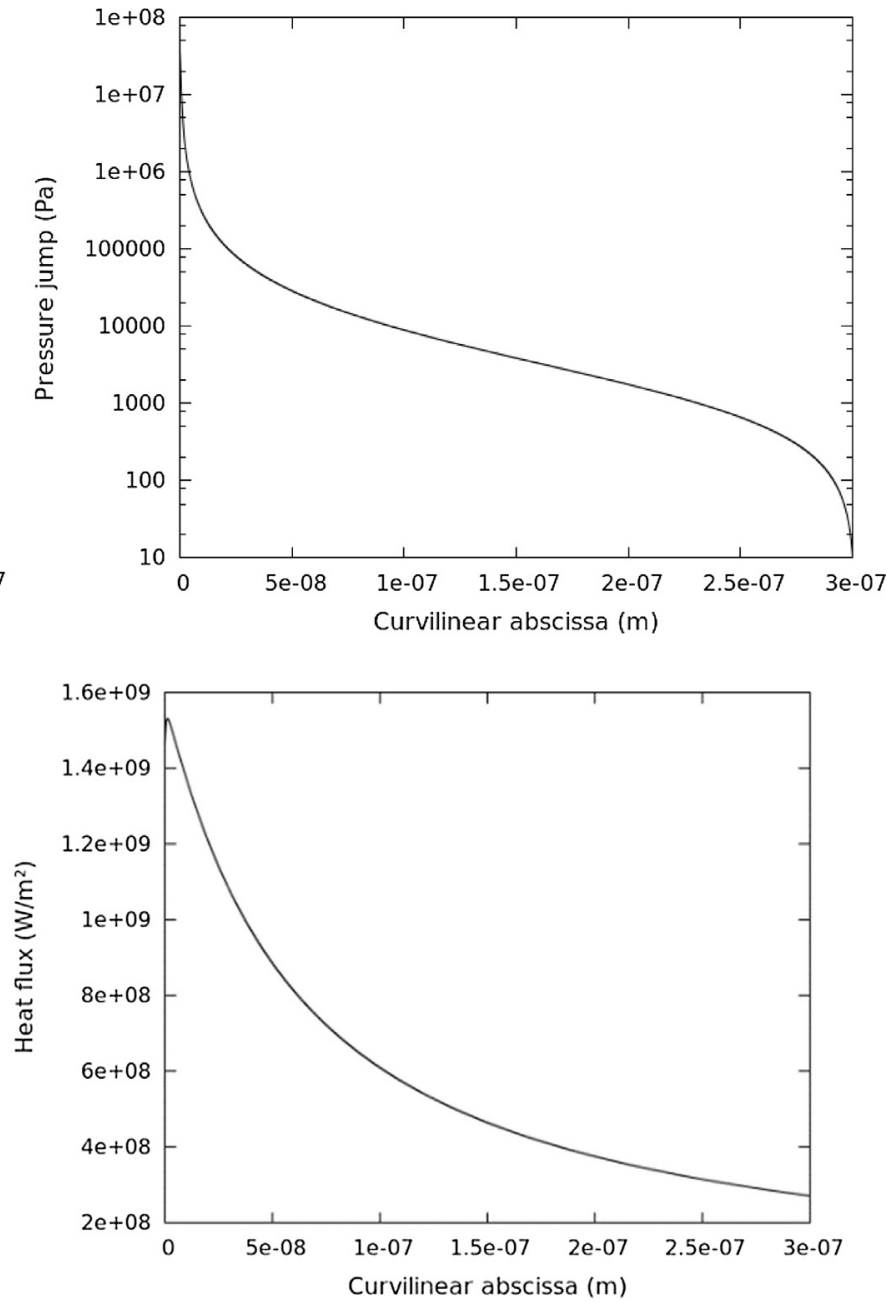

Fig. 3. Angle, pressure jump, integrated heat flux and local heat flux along the interface with the following parameters $\Delta T=100 \mathrm{~K}, \theta_{m i c}=5^{\circ}$. 
physical properties of vapor and liquid phases are indicated in Table 1. A special care has been taken to the spatial convergence of the solution since computations with four successive grids are presented in order to assess that the solution can be independent of the grid size if a sufficiently refined mesh is used. This convergence study will enable us to conclude on the maximum cell size that can be used to perform well-resolved numerical simulations of Nucleate Boiling in the considered configurations. Finally, a numerical test has also been performed to assess the influence of the spatial variation and of the temporal variation of the substrate temperature during the bubble growth on the bubble departure diameter and frequency. In order to achieve this, the thermal solver for two-phase flow has been coupled with an unsteady thermal conduction solver for the solid field at the frontier between the solid phase and the fluid phases. Next an analysis on the temporal evolution of the bubble force balance is presented during its growth in order to clarify the detachment mechanism. The instantaneous pressure and the surface tension are computed all around the bubble and enable the estimation of the different forces acting on the bubble during its growth until its detachment. Different phases of the bubble growth are put into light: a first phase, that is quasi-static with a bubble shape well predicted by integration of the Young-Laplace equation and a dynamic phase just prior the detachment where the dynamic effects does not remain weak. Finally, a parametric study is performed to derive a correlation for the bubble departure radius at different Jakob numbers.

\subsection{Computations of the micro-region model}

Our interest is to compute $\theta_{a p p}, Q_{L}$ for a couple of input parameters $\theta_{\text {micro }}, \Delta T$ by solving the micro region model with the numerical method detailed in Appendix A. We present here results for two couples of parameters, corresponding to two opposite configurations. In the first one, the apparent contact angle strongly differs from the microscopic contact angle, whereas in the second one the two angles have very close values. The physical properties of water used in this study are reminded in Table 1.

Case 1: High wall superheat $(\Delta T=100 \mathrm{~K})$ and low microscopic contact angle $\left(\theta_{\text {micro }}=5^{\circ}\right)$

The values of $\theta_{\text {app }}$ and $Q_{L}$ depend on the chosen value of the integration length $L$. In Fig. 2, we observe that the correct contact angle can be computed by using an integration length $L$ at least equal to $3 \times 10^{-7} \mathrm{~m}$. In Fig. 3 , the angle, the pressure jump, the integrated flux and the local flux are plotted as function of the

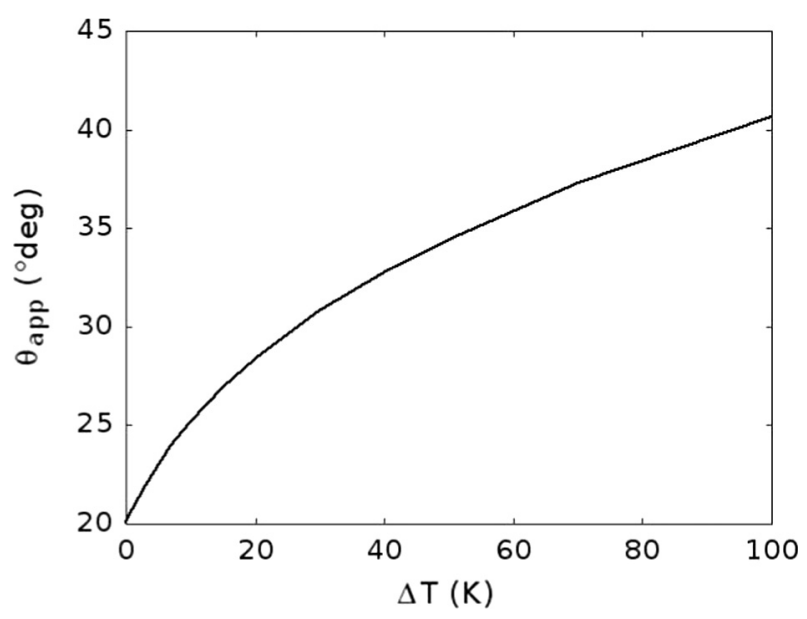

Fig. 5. Apparent contact angle as a function of wall superheat for water with $\theta_{\text {mic }}=20^{\circ}$.

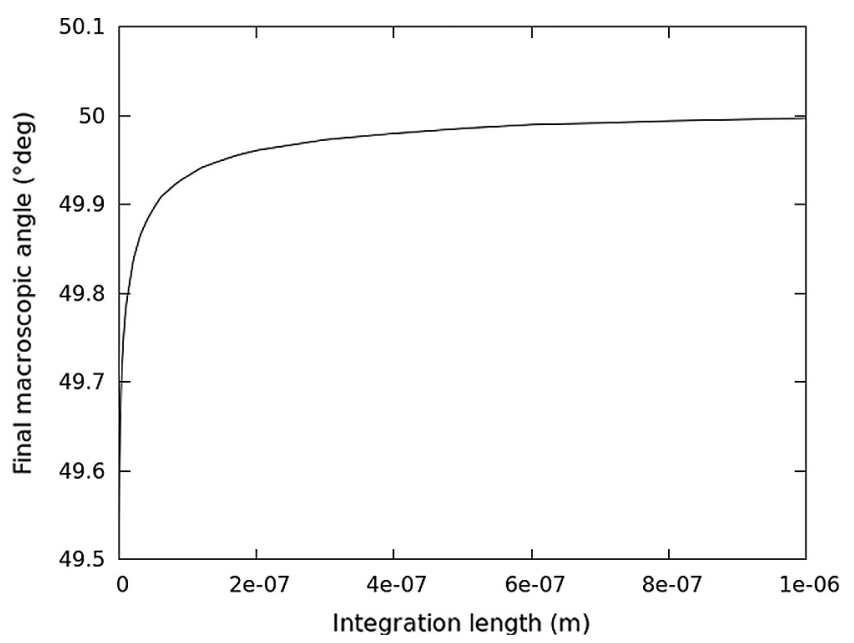

Fig. 6. Apparent contact angle as a function of the total integration length with the following parameters $\Delta T=7 \mathrm{~K}, \theta_{\text {mic }}=49.5^{\circ}$.

curvilinear abscissa. The angle varies from the microscopic contact angle $\left(5^{\circ}\right)$ to the apparent contact angle $\left(39^{\circ}\right)$. In agreement with the pressure jump equation of the micro region model, Eq. (44),
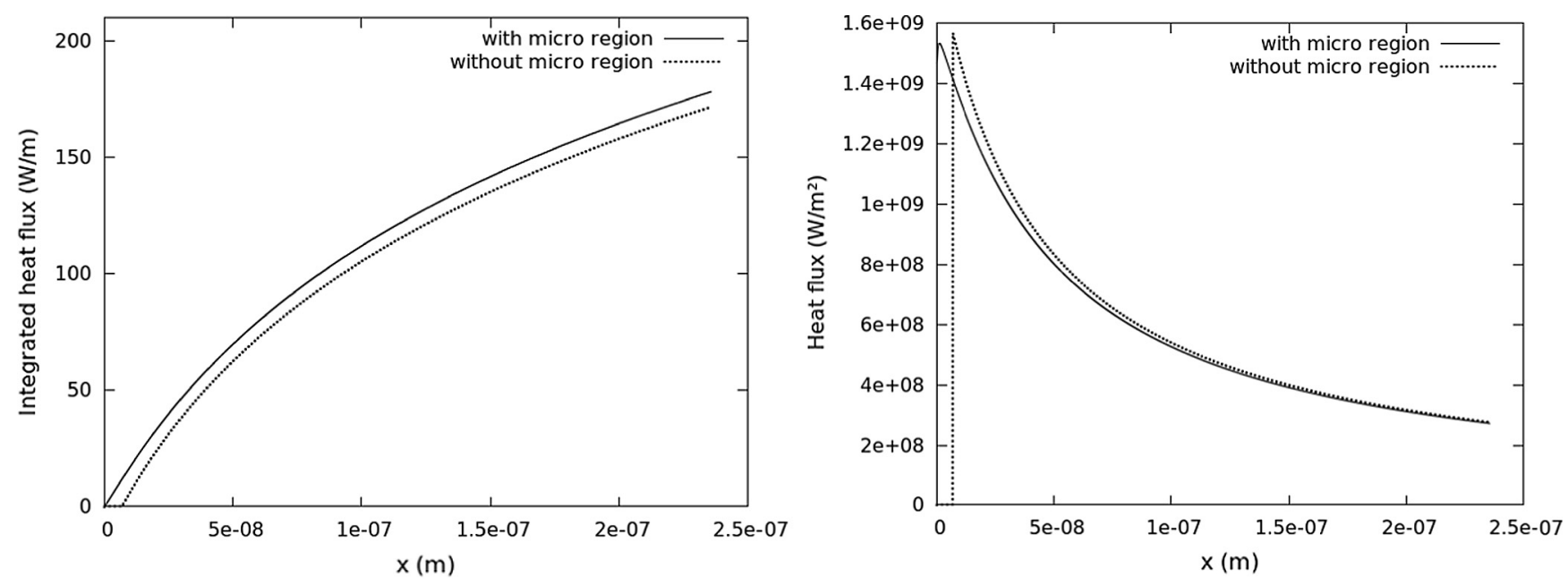

Fig. 4. Integrated heat flux (left) and local heat flux (right) with and without micro region effects with the following parameters $\Delta T=100 \mathrm{~K}, \theta_{m i c}=5^{\circ}$. 

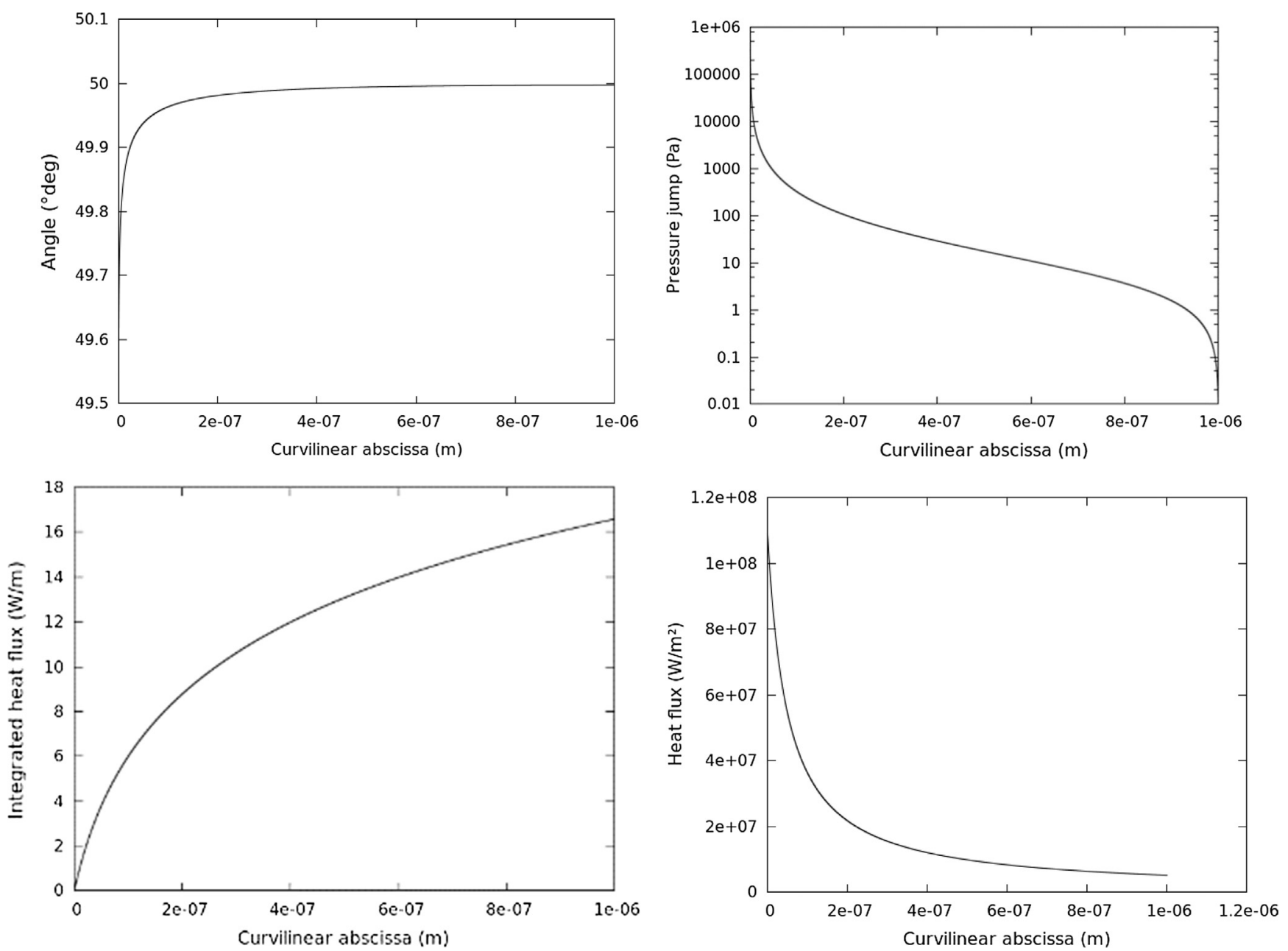

Fig. 7. Angle, pressure jump, integrated heat flux and local heat flux along the interface with the following parameters $\Delta T=7 \mathrm{~K}, \theta_{m i c}=49.5^{\circ}$.
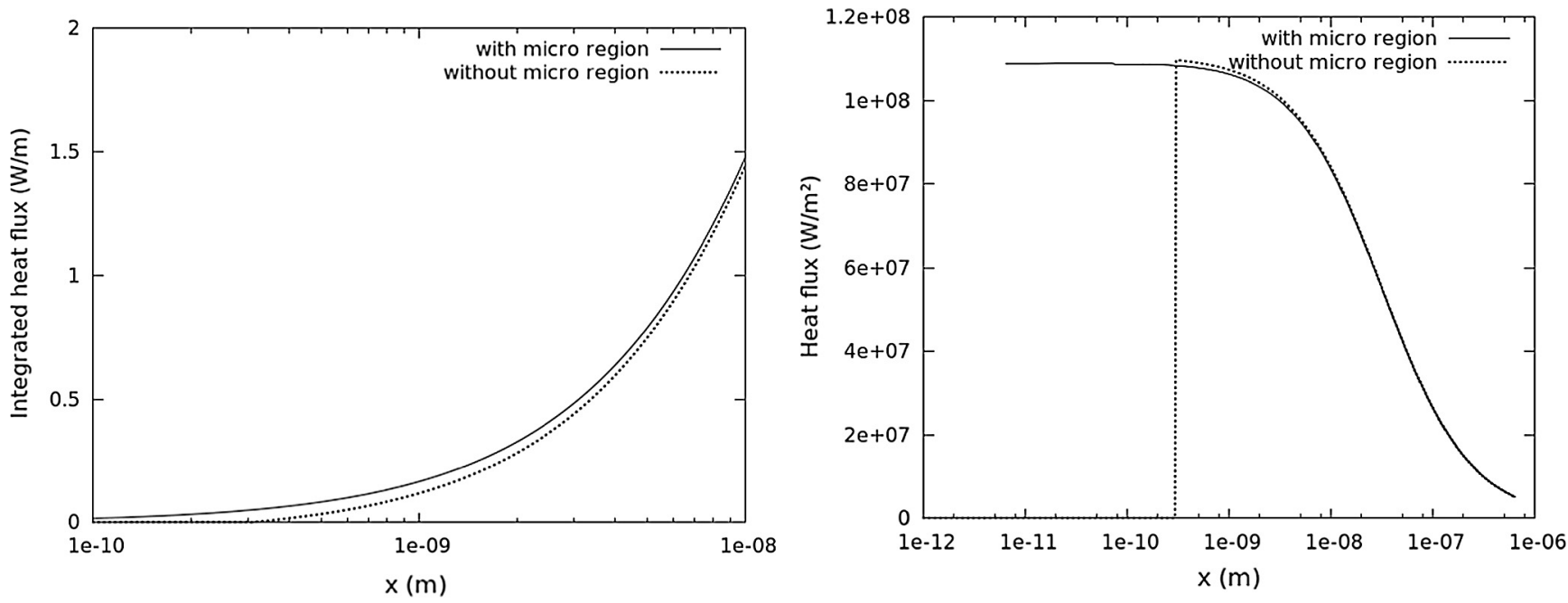

Fig. 8. Integrated heat flux (left) and local heat flux (right) with and without micro region effects with the following parameters $\Delta T=7 \mathrm{~K}, \theta_{m i c}=49.5^{\circ}$.

the pressure jump is proportional to the angle derivative and tends to zero when the curvature becomes zero. Finally, we plot the local heat flux and the integrated heat flux along the curvilinear abscissa, and we compare them with the flux and the integrated flux without the micro-region effects (with constant contact angle equal to $\left.\theta_{\mathrm{app}}\right)$ in Fig. 4. The total flux received by the bubble corresponds to the final value of the integrated flux. When the microregion effects are considered, the evaporation flow is higher due to the increasing interface area. The contribution of the microregion effects on the evaporating flux is computed by the 


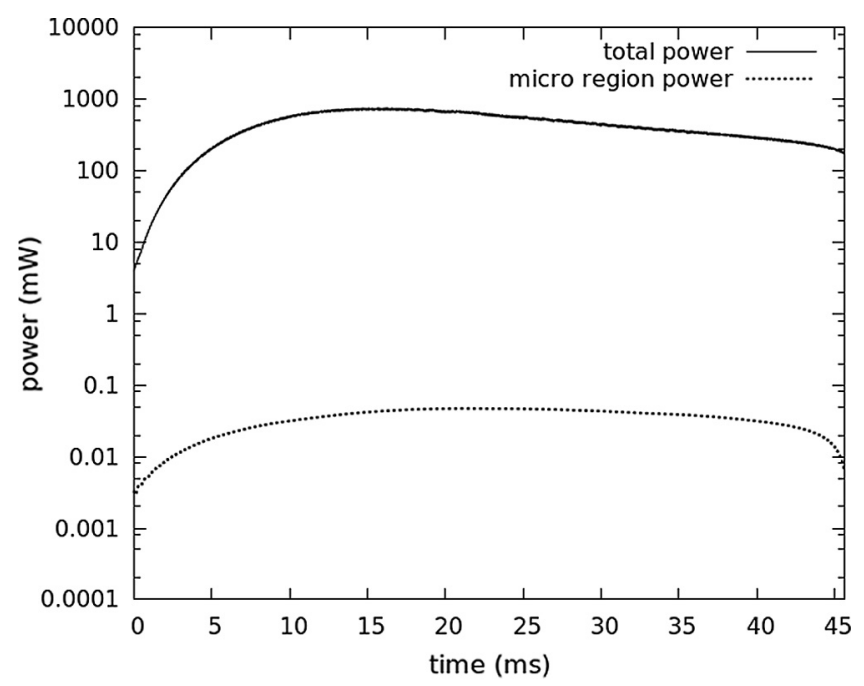

Fig. 9. Comparison between the micro region heat flux and the total heat flux computed by DNS with the following parameters $\Delta T=7 \mathrm{~K}, \theta_{\text {mic }}=49.5^{\circ}$.

difference on the final values of the flux calculated with or without micro-region, i.e., for a constant contact angle. The total contribution on the heat flux of the micro-region is obtained by multiplying this value by the dry spot circumference $2 \pi R_{S}(t)$, where $R_{S}(t)$ is the spreading radius of the bubble along the wall. It is noteworthy that the contribution of the micro-region on the integrated heat flux remains moderate although the gap between the apparent contact angle and the microscopic contact angle is high.

Some additional results are presented in Fig. 5 on the evolution of the apparent contact angle with a wall superheat varying from $0 \mathrm{~K}$ to $100 \mathrm{~K}$, in a configuration involving water and a microscopic contact angle of $20^{\circ}$. For a wall superheat of $100 \mathrm{~K}$, the value of the apparent contact angle is not very different whether a $5^{\circ}$ or a $20^{\circ}$ microscopic contact angle is considered. The latter behavior can be understood considering that the higher is the wall superheat, the lower is the dependence of the apparent contact angle with the microscopic contact angle.

Case 2: Moderate wall superheat $\Delta T=7 \mathrm{~K}$ ) and high microscopic contact angle $\left(\theta_{\text {micro }}=49.5^{\circ}\right)$

This case corresponds to the experiment presented by Son et al. in [52]. The microscopic angle corresponds to an apparent contact angle of $50^{\circ}$ which has been measured in the experiments for a wall superheat $\Delta T=7 \mathrm{~K}$. As shown in Fig. 6, the contact angle will reach a constant value for an integration length of about $1 \mu \mathrm{m}$. Similar to the previous cases, angle, pressure jump, integrated and local flux are plotted in Fig. 7. In this configuration the microscopic contact angle and the apparent contact angle are almost identical, since a small gap of $0.5^{\circ}$ is computed. Consequently, as it is illustrated in Fig. 8, the interface shape and the evaporation flux on the contact line are not modified by micro-region effects in this computation. The contribution of the micro-region on the total heat flux is computed by the difference between the final integrated heat flux with and without micro-region effects multiplied by the dry spot circumference. This contribution is compared in Fig. 9 to the total heat flux received by the vapor bubble computed by the Direct Numerical Simulation, presented in the next section, without using any micro-region model. From this figure, we can observe that the micro-region heat flux is more than 10,000 times smaller than the total heat flux computed with the Direct Numerical Simulation.

From these preliminary results, we can assume that, in the specific case considered here, i.e. a moderate wall superheat, a high microscopic contact angle and high density ratio, the micro-region heat flux will be negligible. This result will be confirmed in the next section, by the comparisons between experimental data and Direct Numerical Simulations of Nucleate Boiling without using any specific micro-region model of the heat flux in the vicinity of the contact line. It should be mentioned here, that the apparent contact angle tends to the same value that the microscopic contact angle for moderate wall superheat and high microscopic contact angle, and it allows to explain why the micro-region flux can be neglected in this case. But as illustrated previously in other configurations, with higher wall superheat and lower microscopic contact or perfectly wetting fluids, this assumption might not be valid anymore. Moreover, our study is limited to high density ratio between the two phases, since the physical properties of water at ambient pressure are used in the simulations. Therefore, as the influence of the density ratio has not been investigated either, more extensive studies will be required in configurations with lower density ratio, as it can be the case for applications involving higher pressure, for instance.

In Fig. 10, a parametric study on the evolution of the apparent contact angle versus the microscopic contact angle is presented for a wall superheat $\Delta T=7 \mathrm{~K}$. From this figure, it can be observed that for a microscopic contact angle below $30^{\circ}$, the apparent
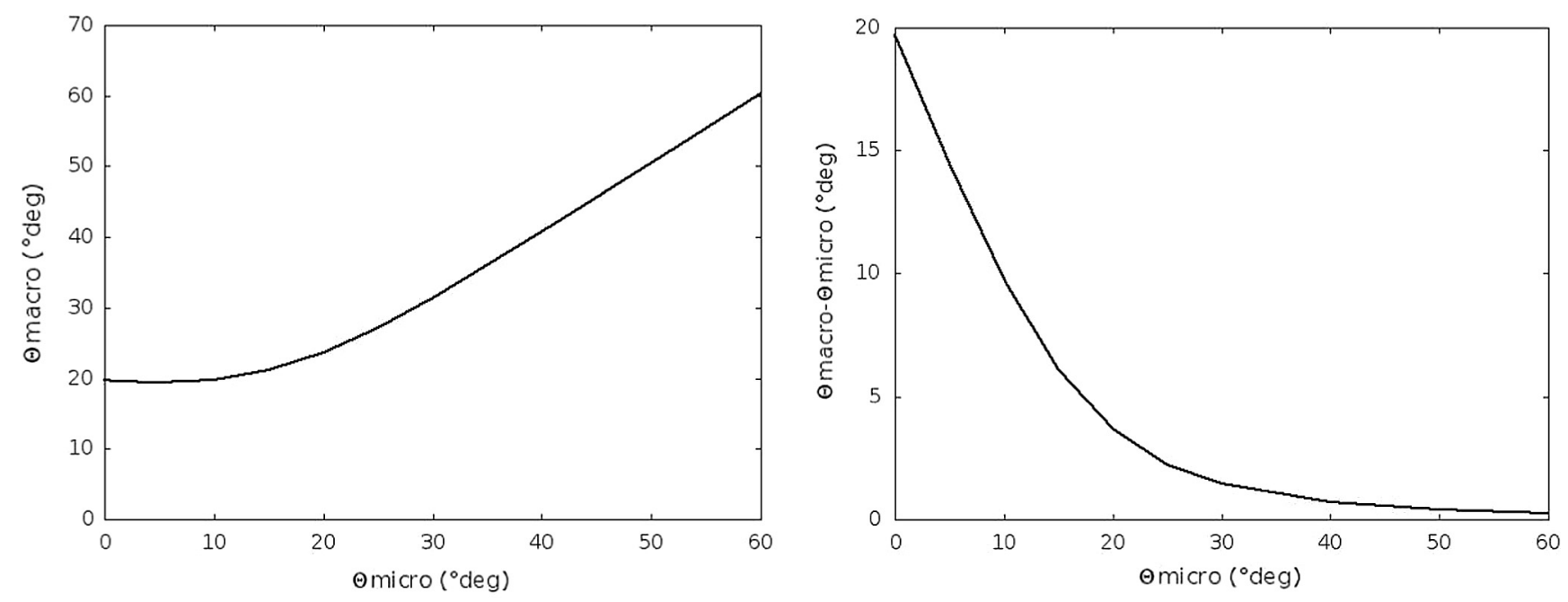

Fig. 10. Apparent contact angle as a function of the microscopic contact angle with the following parameters $\Delta T=7 \mathrm{~K}$. 


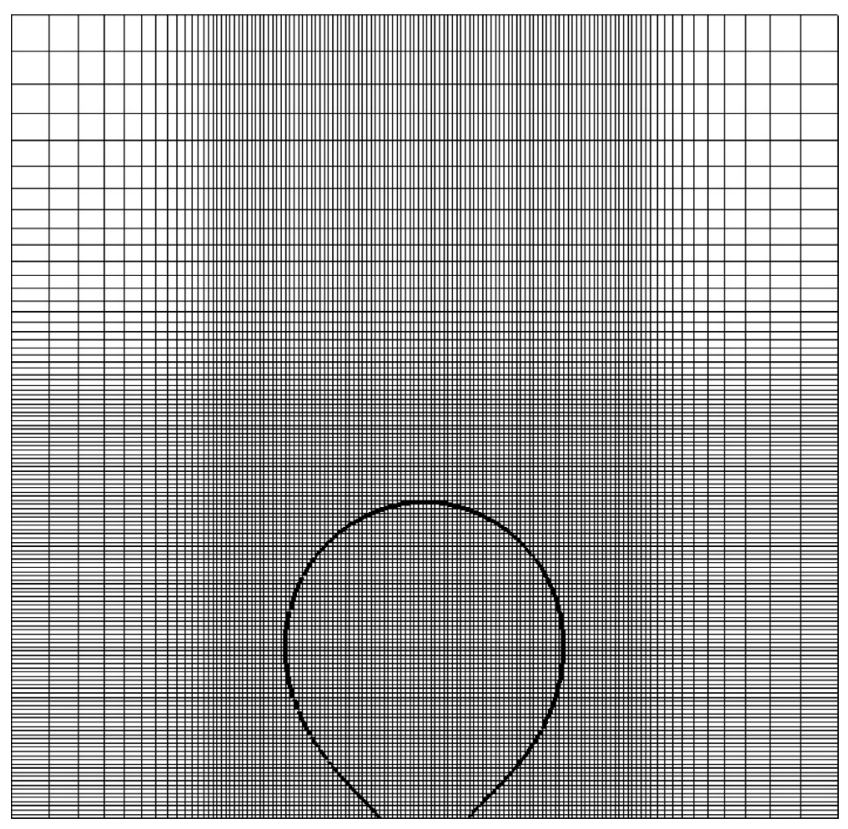

Fig. 11. Visualization of a non-uniform coarse grid $(64 \times 128)$ and of the interface position before the bubble detachment.

contact angle is still superior to $20^{\circ}$ and it slowly varies with the microscopic contact angle, whereas for microscopic contact angle above $30^{\circ}$ the difference between the microscopic contact angle and the apparent contact angle is low and tends to 0 .

Considering the numerical results presented in this preliminary study on the micro-region heat flux, carried out with the model proposed by Mathieu et al in [35,36] for partially wetting liquid, we will assume in the rest of this paper that the micro-region effects are negligible in configurations involving both a moderate wall superheat and a high microscopic contact angle for applications at ambient pressure. Since just a slight difference exists between the microscopic contact angle and the macroscopic contact angle the heat flux contribution is very low in comparison to the heat flux already computed by DNS. Therefore, in the simulations presented in the previous section, we will not include any micro-region model in the vicinity of the contact line.

\subsection{Validations of the direct numerical simulation of nucleate boiling}

In this section, we present two benchmarks for the validation of a bubble growth on a superheated wall, such as it has been firstly proposed in [52] with the following parameters $J a=21$ and $J a=25.5, \theta_{\text {app }}=50^{\circ}$ and a density ratio $\rho_{\text {liq }} / \rho_{\text {vap }}=1604$. The simulation results are compared with experimental data. The experimental set-up consists in a hot wall immersed in a water pool whose temperature is equal to the saturation temperature at atmospheric pressure. Two values of the wall superheat have been used, i.e. $\Delta T=7 \mathrm{~K}$ and $\Delta T=8,5 \mathrm{~K}$ corresponding respectively to Jakob numbers $\mathrm{Ja}=21$ and $\mathrm{Ja}=25.5$. The wall is supposed to be thick enough to consider that its temperature is uniform. The relevance of this assumption will be discussed more deeply in a next part. The simulations are carried out in an axisymmetric configuration in a computational box whose dimensions are $l_{r}=4 \mathrm{~mm}$, $l_{z}=8 \mathrm{~mm}$. Whatever the number of computational cells, the mesh is divided into two parts, a uniform one in the region where the bubble is attached to the wall and a non-uniform one in the rest of the domain. This decomposition in two regions enables to lower the total number of grid cells while maintaining the boundary conditions far from the bubble in order to reduce the containment effects. A typical grid is plotted in Fig. 11 where the uniform region is a $2 \mathrm{~mm} \times 4 \mathrm{~mm}$ rectangle shape. Four successive grids have been used with the following cell length of $40 \mu \mathrm{m}, 20 \mu \mathrm{m}, 10 \mu \mathrm{m}$ and $5 \mu \mathrm{m}$ in the uniform refined zone which correspond respectively to the following meshes $64 \times 128,128 \times 256,256 \times 512$, $512 \times 1024$.

The initial thermal field is uniform and equal to the saturation temperature in the whole domain, except in a thermal boundary layer close to the wall. This thermal boundary layer results from the free convection above a horizontal plate. We use the following correlation from Kays and Crawford [23] to express $\delta$, the thickness of this boundary layer

$\delta=7.14\left(\frac{\mu_{l} \alpha_{l}}{g \beta_{T} \Delta T}\right)^{\frac{1}{3}}$,

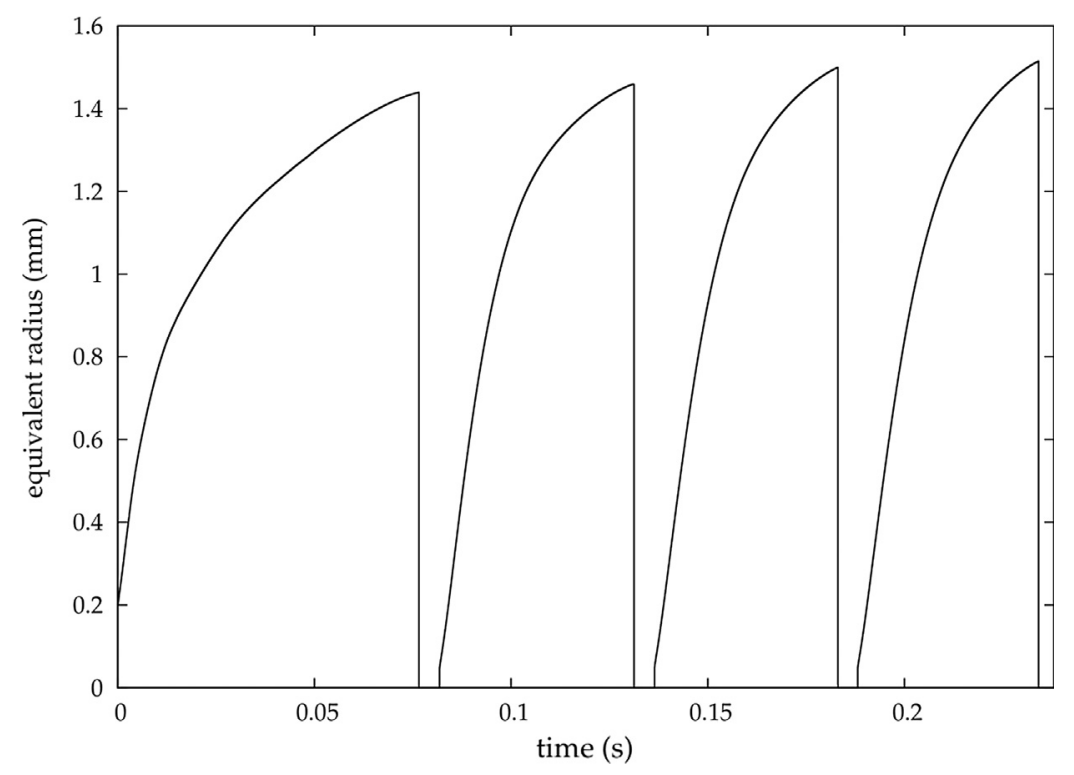

Fig. 12. Temporal evolution of the bubble radius during the first four bubble detachment cycles. 

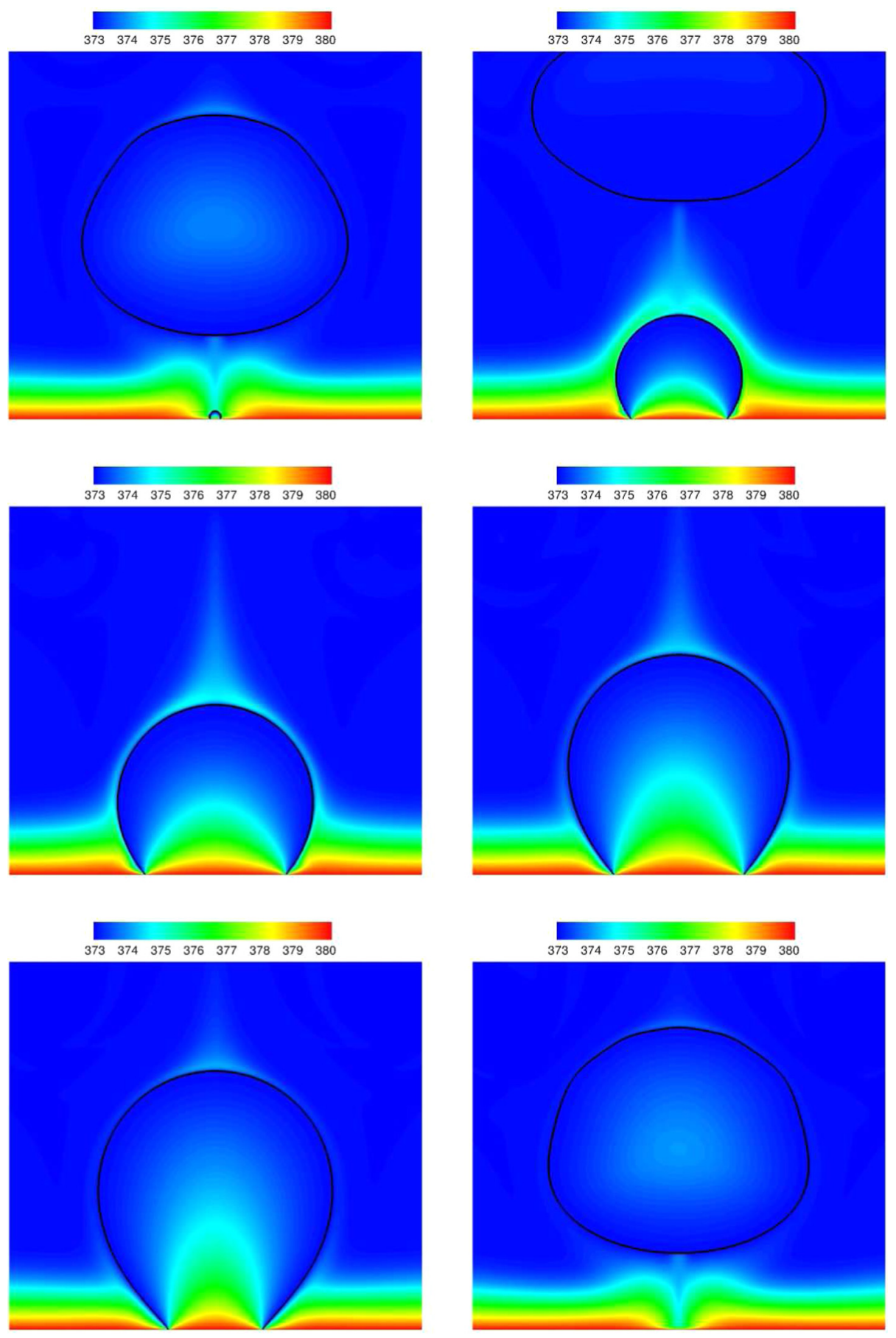

Fig. 13. Temperature field and interface position at different times of a bubble growth cycle with the following parameters $\Delta T=7 \mathrm{~K}, \theta_{a p p}=50^{\circ}$.

with $\beta_{T}$ the coefficient of thermal dilatation at constant pressure, and $\alpha_{l}$ the thermal diffusivity of the liquid. From this expression, a linear temperature profile is imposed to describe the thermal boundary layer. The bubble is initialized as a half sphere nucleus whose initial radius is equal to $60 \mu \mathrm{m}$.

Once the bubble has grown and detached due to the buoyancy force, a new nucleus is artificially generated in order to compute the growth of the next bubble. A transient regime is observed during the first two bubble departures. Indeed, the bubble diameter departure and the bubble frequency departure are varying until the third detachment is reached, and next they remain constant for the following cycles, such as it can be visualized in Fig. 12 . The temperature field and the interface position have been plotted in Fig. 13 for different times of an entire cycle of the bubble growth. In Fig. 14, the temporal evolution of the bubble radius has been plotted with the four different grids during the growth of the first computed bubble. This figure clearly brings out the spatial convergence of the computation from the grid $256 \times 512$ corresponding to a mesh length equal to $10 \mu \mathrm{m}$. Next, for two values of the Jakob number, comparisons between our numerical simulations and experiments from [52] are presented in Figs. 15 and 16 on the temporal evolution of the bubble radius in the stabilized regime 


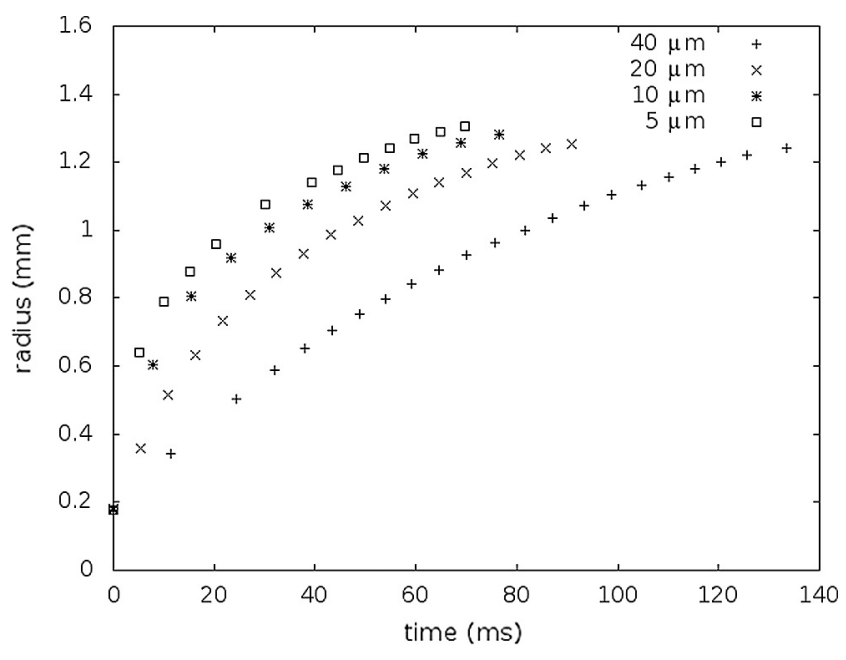

Fig. 14. Temporal evolution of the bubble radius for different sizes of the grid cells for the first bubble detachment with the following parameters $\Delta T=7 \mathrm{~K}, \theta_{a p p}=50^{\circ}$.

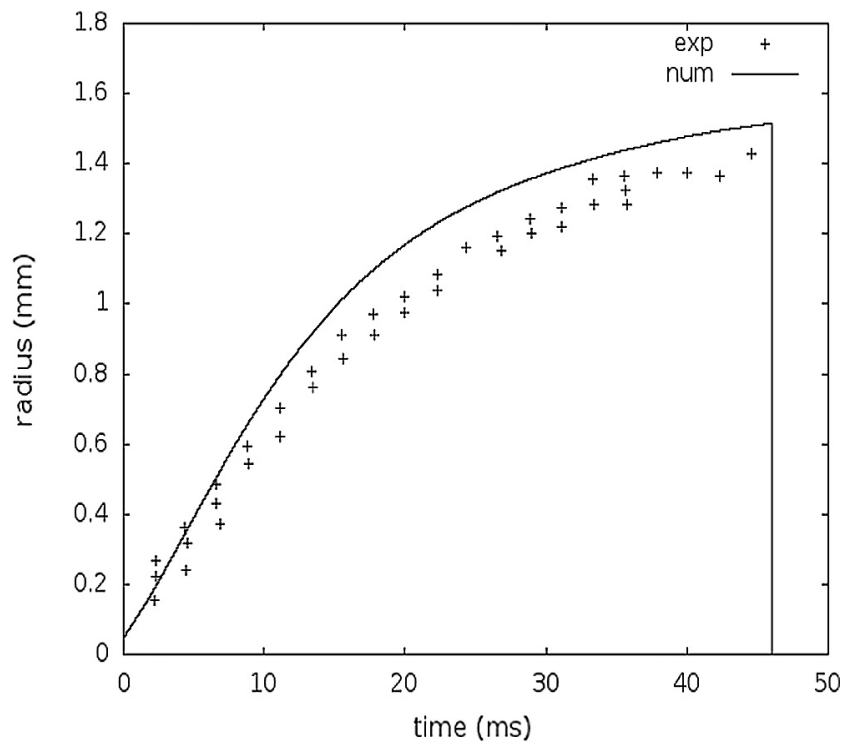

Fig. 15. Comparison on the temporal evolution of the bubble radius between the numerical simulation $(\Delta z=\Delta r=10 \mu \mathrm{m})$ and experimental data for a wall superheat $\Delta T=7 \mathrm{~K}$ and an apparent contact angle $\theta_{\text {app }}=50^{\circ}$.

(after the third departure). These figures show a good agreement of the numerical simulations with the experimental data. The gap between the numerical predictions and the experimental results, reported in Table 2, is less than ten per cent, both on radius detachment and frequency detachment. It is noteworthy that more important discrepancies between numerical simulations and measurements are usually reported, in the difficult context of nucleate boiling. For instance in [26], the authors claim that a level of error of about twenty per cent was usual in that kind of configurations

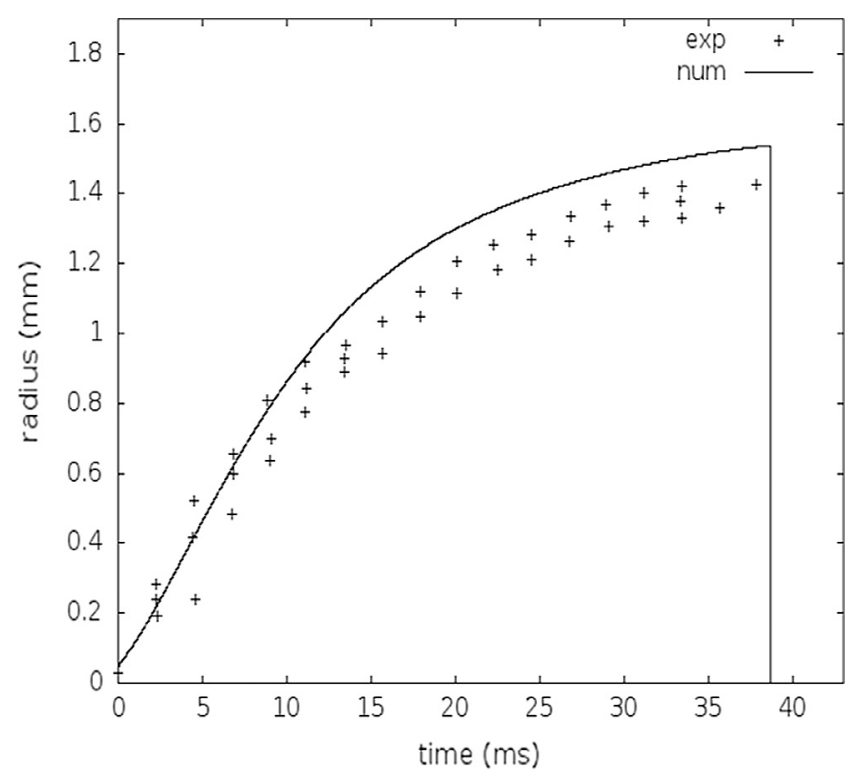

Fig. 16. Comparison on the temporal evolution of the bubble radius between the numerical simulation $(\Delta z=\Delta r=10 \mu \mathrm{m})$ and experimental data for a wall superheat $\Delta T=8.5 \mathrm{~K}$ and an apparent contact angle $\theta_{\text {app }}=50^{\circ}$.

where many uncertainties can affect the comparison between the numerical solution and the experimental results. Finally to elucidate the influence of the wall temperature variation in the vicinity of the contact line, we also present some results of numerical simulations accounting for the heat conduction in the solid substrate by coupling an unsteady heat conduction solver with our overall two-phase flow solver. The wall is made of silicon and is $1.3 \mathrm{~mm}$ thick. The properties of silicon are the following: $k_{s}=148 \mathrm{~W} \mathrm{~m}^{-1}$ $\mathrm{K}^{-1}, C p_{s}=700 \mathrm{~J} \mathrm{~K}^{-1} \mathrm{~kg}^{-1}$ and $\rho_{s}=2330 \mathrm{~kg} \mathrm{~m}^{-3}$, where $k_{s}$ is the thermal conductivity, $C p_{s}$ the thermal capacity and $\rho_{s}$ the density of silicon. As very low time steps must be imposed in two-phase flows simulations in order to respect the stringent temporal constraint due to capillary effects, a simple explicit coupling has been carried out between the two domains. It means that each subdomain is solved separately by using the previous solution of the other subdomain to impose the time-varying boundary condition at the frontier between the two domains. At the bottom boundary, a thermal flux is imposed with a Neumann boundary condition on the temperature derivative both to ensure the thermal flux continuity between the solid domain and the fluid domain and to impose the attended wall temperature. This thermal flux is expressed as follows,

$\varphi_{\text {bottom }}=\frac{k_{l}\left(T_{\text {wall }}-T_{\text {sat }}\right)}{\delta}$.

Inside the solid domain, the temperature field wall is initialized by a linear profile corresponding to the thermal flux which is imposed with Eq. (47).

In Fig. 17, the thermal field is plotted at two different times of the bubble history, when the contact line is advancing and when the contact line is receding a few time before the bubble

Table 2

Relative difference between the experimental data and the numerical simulation on the bubble radius departure $\varepsilon_{r}$ and on the frequency departure $\varepsilon_{f}$.

\begin{tabular}{|c|c|c|c|c|}
\hline & $\varepsilon_{r}(\Delta T=7 \mathrm{~K})(\%)$ & $\varepsilon_{f}(\Delta T=7 \mathrm{~K})(\%)$ & $\varepsilon_{r}(\Delta T=8.5 \mathrm{~K})(\%)$ & $\varepsilon_{f}(\Delta T=8.5 \mathrm{~K})(\%)$ \\
\hline$\Delta z=\Delta r=40 \mu \mathrm{m}$ & -2.10 & -64.2 & -1.4 & -65.7 \\
\hline$\Delta z=\Delta r=20 \mu \mathrm{m}$ & +2.10 & -33.3 & $+3.23 \%$ & -35.3 \\
\hline$\Delta z=\Delta r=10 \mu \mathrm{m}$ & +5.94 & -4.95 & +7.78 & +3.59 \\
\hline
\end{tabular}



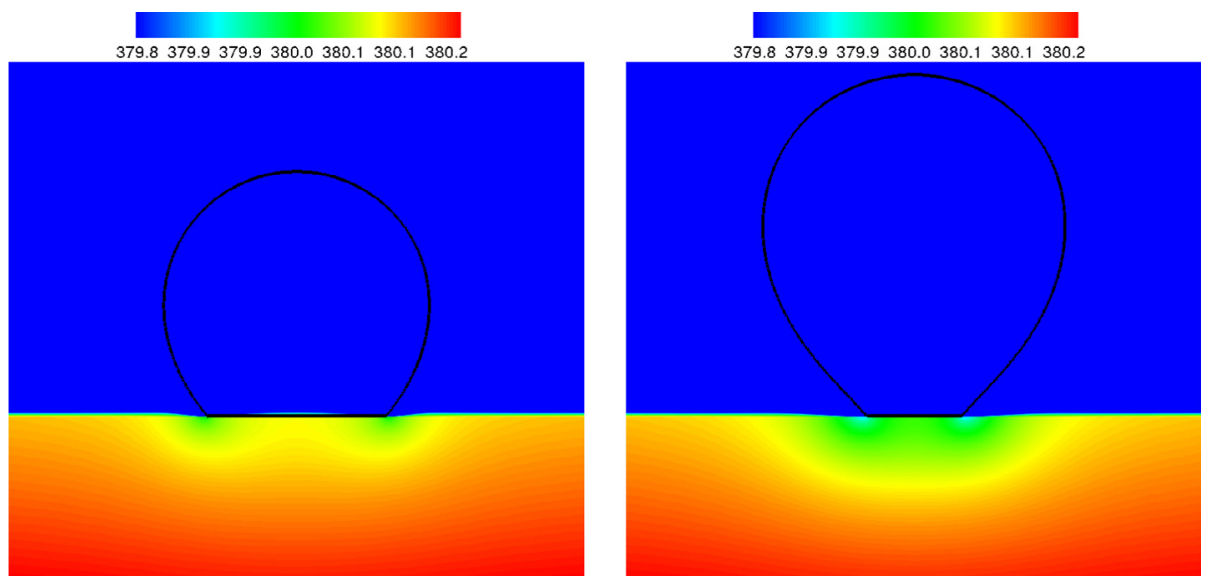

Fig. 17. Interface position and temperature field inside the solid wall at different times.

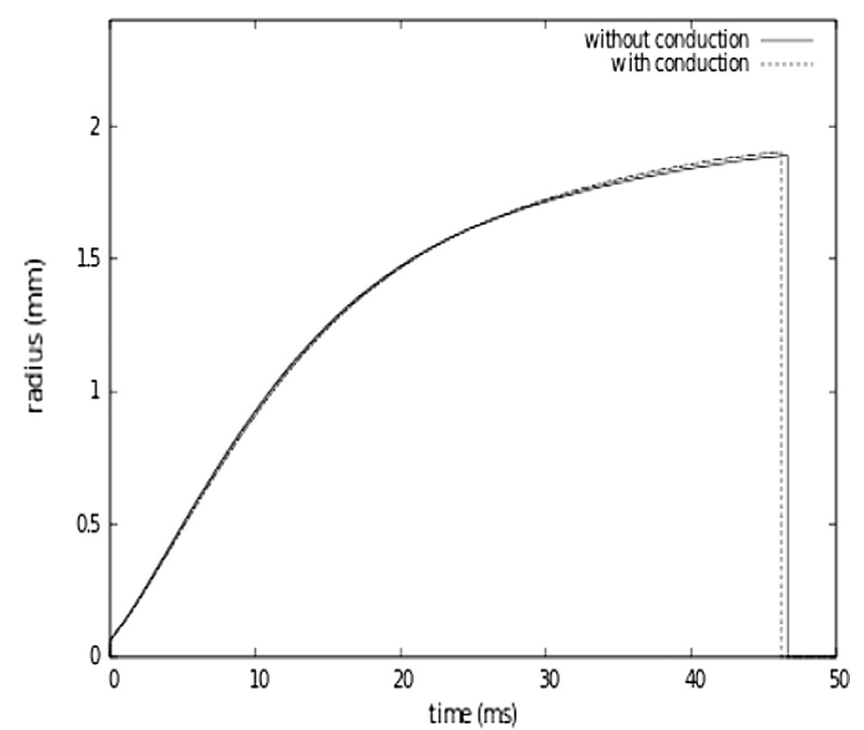

Fig. 18. Comparison on the temporal evolution of the bubble radius between two numerical simulations with or without the computation of the heat conduction inside the hot substrate with the following parameters $\Delta z=\Delta r=10 \mu \mathrm{m}$ and $\Delta T=7 \mathrm{~K}$.

detachment. It can be observed from these figures that weak temperature variations (less than $0.2 \mathrm{~K}$ ) occur along the frontier between the two domains during the bubble growth. Finally, we present in Fig. 18 a comparison between two computations, one involving a solid heat conduction solver and the other using an isothermal boundary condition on the wall. It demonstrates that, in the considered configuration, the high thermal conductivity and diffusivity quickly homogenize the temperature field in the solid and the temporal evolution of the bubble radius is very similar to those obtained with an isothermal boundary condition on the wall.

This preliminary part allows us concluding on the effectiveness of the numerical solver that we have developed to perform Direct Numerical Simulation of Nucleate Boiling. Many important points have been addressed in these computations, as the minimal size of the computational cell which should be close to $10 \mu \mathrm{m}$ to converge and on the suitability of an isothermal boundary condition on the wall in the considered configuration, which corresponds to a low Biot number in the solid domain. Moreover, the comparison between numerical results and experimental results confirm the hypothesis that we have formulated in the previous Section 4.1. Indeed, it has been shown that the numerical simulation converges to a solution in good agreement with experiments, without using any micro region model. It confirms that the micro region effects are negligible, in the vicinity of the contact line, for configurations involving high static contact angle (up to $30^{\circ}$ ), a moderate wall superheat and a high density ratio.

\subsection{Force balance during the bubble growth}

We now study the temporal evolution of the mechanical force balance acting during the bubble growth in order to improve our physical understanding of the bubble departure by a more deepened analysis on the simulation presented in Section 2 with the physical properties of water and the following parameters $\Delta T=7 \mathrm{~K}, \theta_{a p p}=50^{\circ}$. The temporal evolution of the equivalent radius, of the spreading radius of the bubble foot, of the energy flux received by the bubble and the added mass force are plotted in Fig. 19.

An image sequence is plotted in Fig. 20 to visualize the temporal evolution of the bubble shape computed by DNS. This sequence is compared with the static bubble equilibrium shape model corresponding to the same bubble volume. The latter has been computed by integrating the Laplace equation with the same volume and contact angle in a hydrostatic pressure field. It is well known that this static model has no solution when the bubble radius reaches a value above a critical radius known as the Fritz radius in [10], and defined with the following relation

$R_{F}=0.0104 \theta \sqrt{\frac{\sigma}{g\left(\rho_{L}-\rho_{V}\right)}}$.

This critical radius corresponds to the maximum radius value of an attached bubble before departure from the static mechanical equilibrium. As the bubble radius is above the Fritz radius on the last two pictures in Fig. 20, the bubble shape, computed with DNS, can no longer be compared with the theoretical static equilibrium bubble shape.

Plotted in Fig. 21 is the temporal evolution of the forces acting on the bubble during the first cycle, i.e. the static forces (Archimede force, the contact pressure force, and the capillary force) and the dynamical force (added mass which is equal to the opposite to the sum of the static forces). In comparison with the other forces, the weight of the bubble is neglected due to the low vapor density. A detailed expression of all these forces is provided in Appendix B. The evolution of the pressure field is plotted in Fig. 22. By analyzing all these data, we have distinguished six steps 


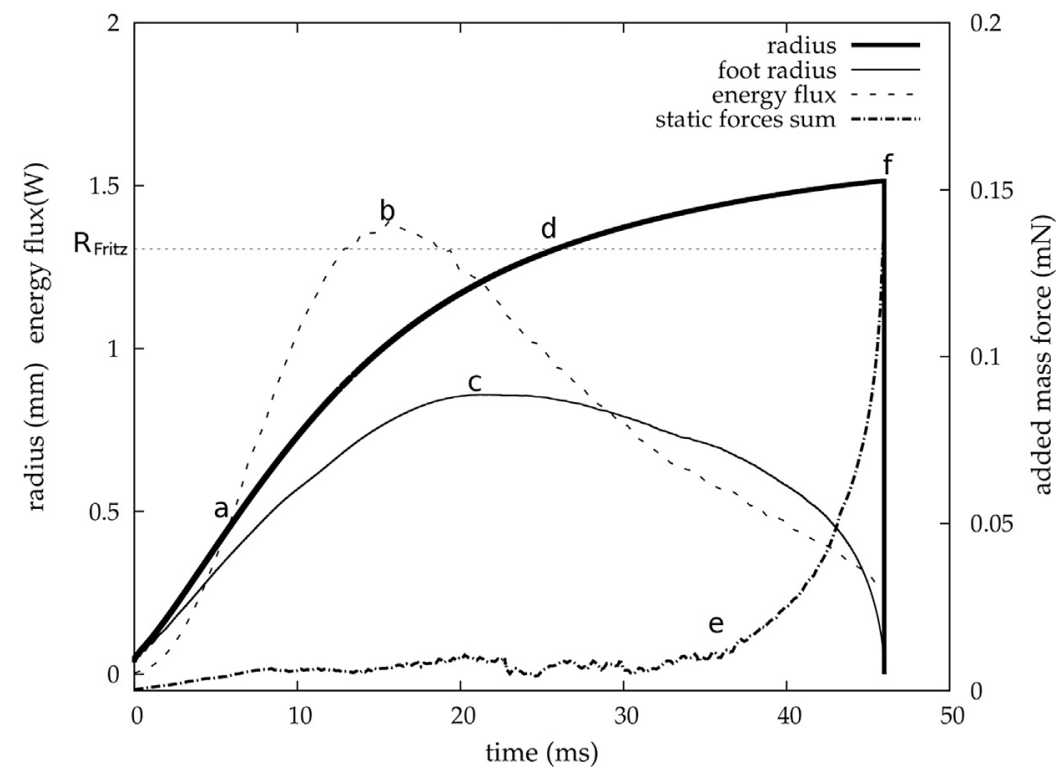

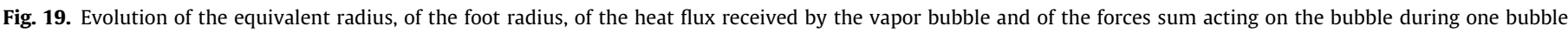
growth cycle.

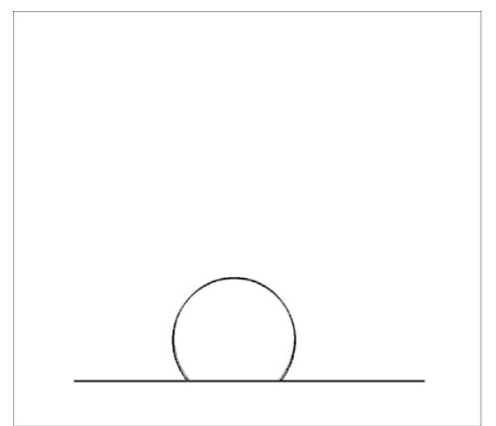

A

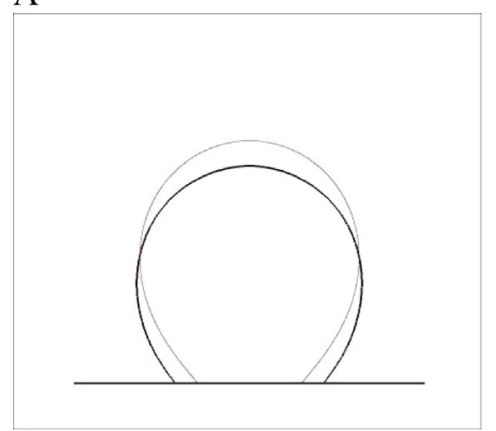

$\mathrm{D}$

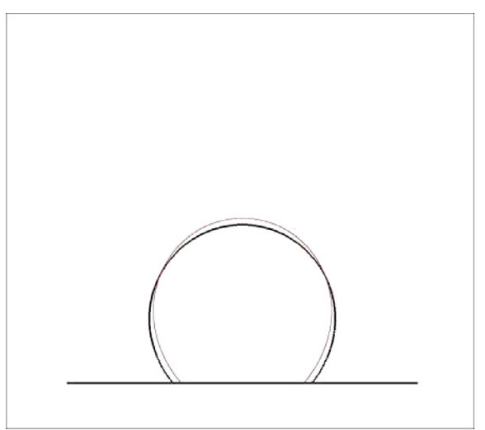

B

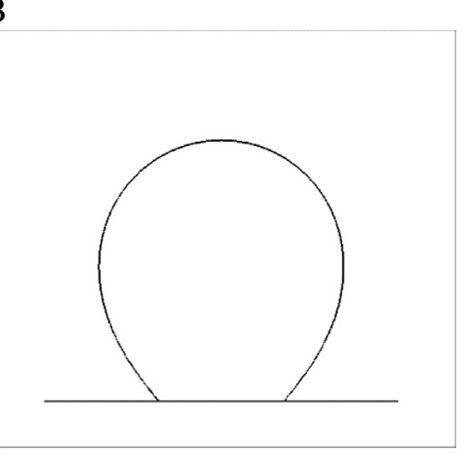

E

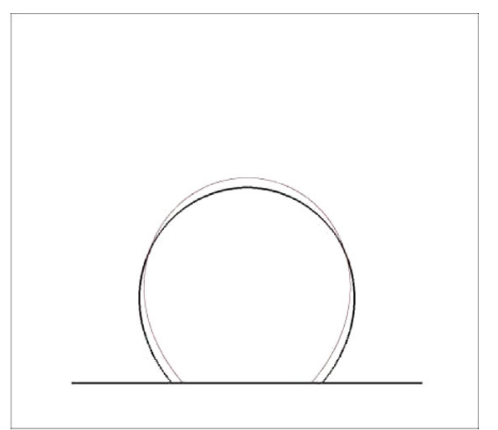

C

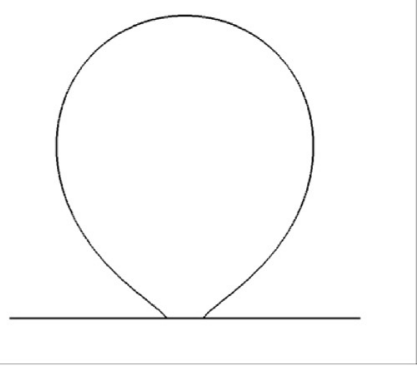

F

Fig. 20. Comparison between the shape of the bubble computed by DNS (black line) and the bubble computed using the static force balance model (grey line).

during the bubble growth, which are respectively flagged by the letter a, b, c, d, e, f, in Fig. 19, and which are detailed hereafter:

a. Due to evaporation the volume of the water vapor bubble is increasing. The bubble shape is similar to the static bubble one.

b. The heat flux received by the bubble is maximum. It leads to an inflexion point on the equivalent radius evolution. Next, the bubble growth rate will decrease. While the added mass force is close to zero, the bubble growth is quasi-static in accordance to the Laplace law (Appendix B). The bubble shape computed by the numerical simulation are similar to the shapes computed with the static equilibrium model. c. The bubble is still growing and the spreading radius reaches its maximum. It does not correspond to a breakdown of the equilibrium static state. At this time, the wall pressure reaction and the capillarity force are maximum, as it can visualised in Fig. 21.

d. The bubble equivalent radius reaches the Fritz radius. Nevertheless, the sum of the forces shows that the bubble is still at equilibrium state on the wall. Indeed, the bubble growth is no longer quasi-static, and the added mass force, due to the liquid inertia around the bubble, is also acting on the bubble. This force is not considered in the calculation of the Fritz radius, and it may retain the bubble to the wall, and delay the bubble detachment. 


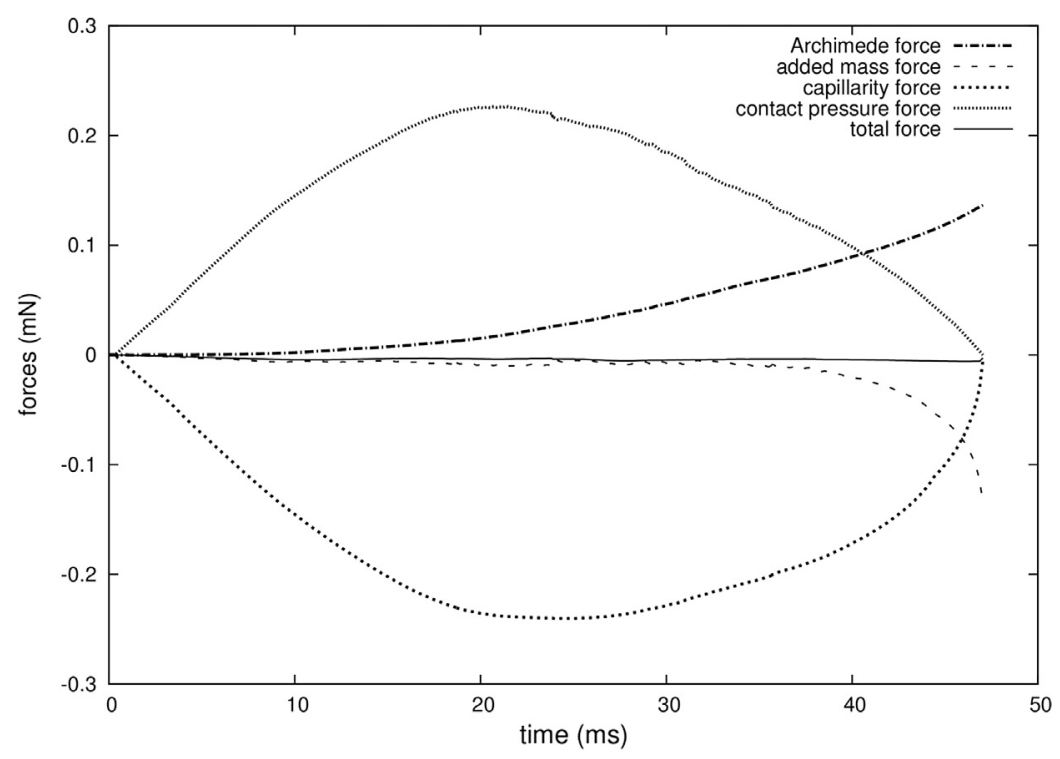

Fig. 21. Temporal evolution of the forces acting on the bubble during one growth cycle.

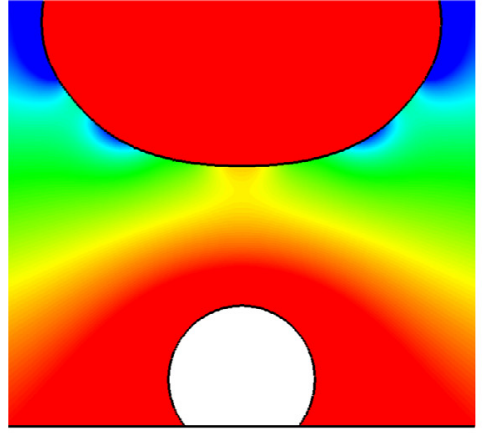

A

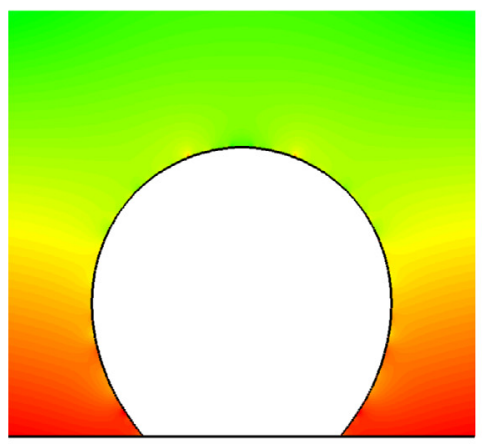

D

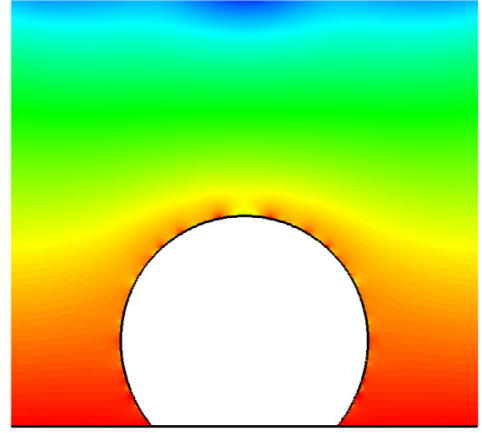

B

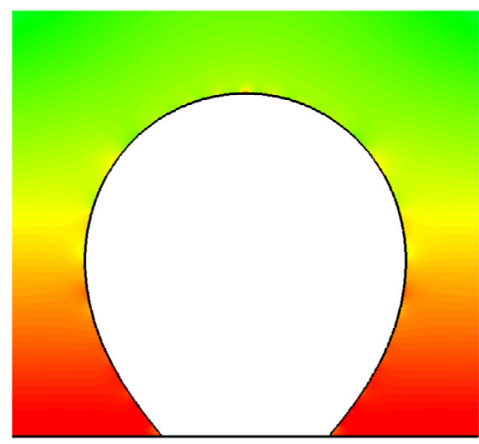

E

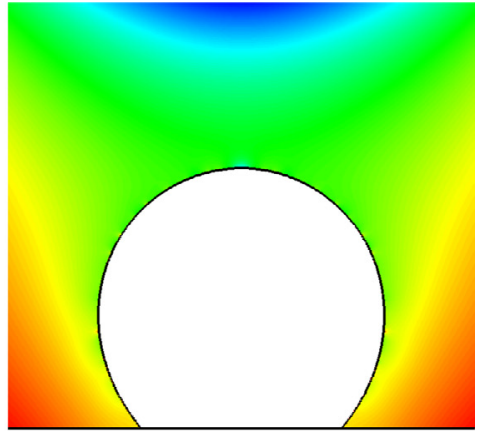

C

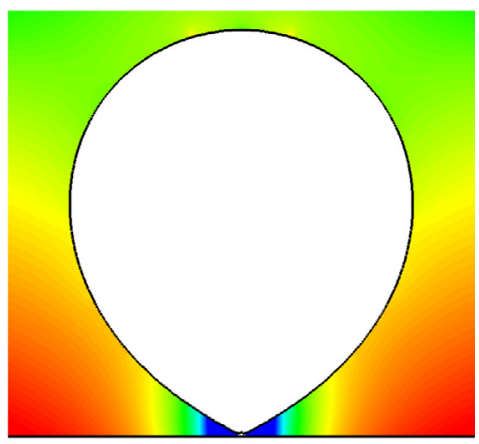

$\mathrm{F}$

Fig. 22. Pressure field evolution during one bubble growth cycle.

e. As the bubble is still growing, the static equilibrium can no longer be respected. The added mass force is emerging, allowing to close the forces balance to zero. The bubble starts to leave the nucleation site.

f. The foot radius tends to zero. When the bubble is detaching, we observe a pressure drop in the vicinity of the detachment point due to the liquid inertia during the bubble pinch-off.

\subsection{Departure radius correlation}

Based on the analysis, proposed in the previous section, we can deduce that the departure radius $R_{d e p}$ is larger than the radius given by Fritz correlation. This leads to the following general expression of the dimensionless bubble departure radius,

$\frac{R_{\text {dep }}}{R_{\text {Fritz }}}=1+\gamma$. 
Table 3

Variation of the parameters in the simulations reported in Fig. 22.

\begin{tabular}{|c|c|c|c|c|c|}
\hline $\mathrm{Ja}$ & $\Delta \mathrm{T}(\mathrm{K})$ & $\mathrm{L}_{\mathrm{V}}\left(\mathrm{J} \mathrm{kg}^{-1}\right)$ & $\mathrm{R}_{\text {dep }} / \mathrm{R}_{\text {Fritz }} \mathrm{DNS}$ & $\mathrm{R}_{\text {dep }} / \mathrm{R}_{\text {Fritz }}$ Correlation & Relative error (\%) \\
\hline 10.49 & 7 & $4,513,000$ & 1.0656 & 1.0631 & -0.233 \\
\hline 11.98 & 4 & $2,256,000$ & 1.0736 & 1.0764 & 0.0026 \\
\hline 16.48 & 6 & $2,256,000$ & 1.1157 & 1.1204 & 0.0042 \\
\hline 20.97 & 7 & $2,256,000$ & 1.1723 & 1.1700 & -0.0019 \\
\hline 25.47 & 9 & $2,256,000$ & 1.2246 & 1.2244 & -0.0002 \\
\hline 29.96 & 10 & $2,256,000$ & 1.2836 & 1.2831 & -0.0004 \\
\hline 41.95 & 7 & $1,128,250$ & 1.4544 & 1.4580 & 0.0025 \\
\hline
\end{tabular}

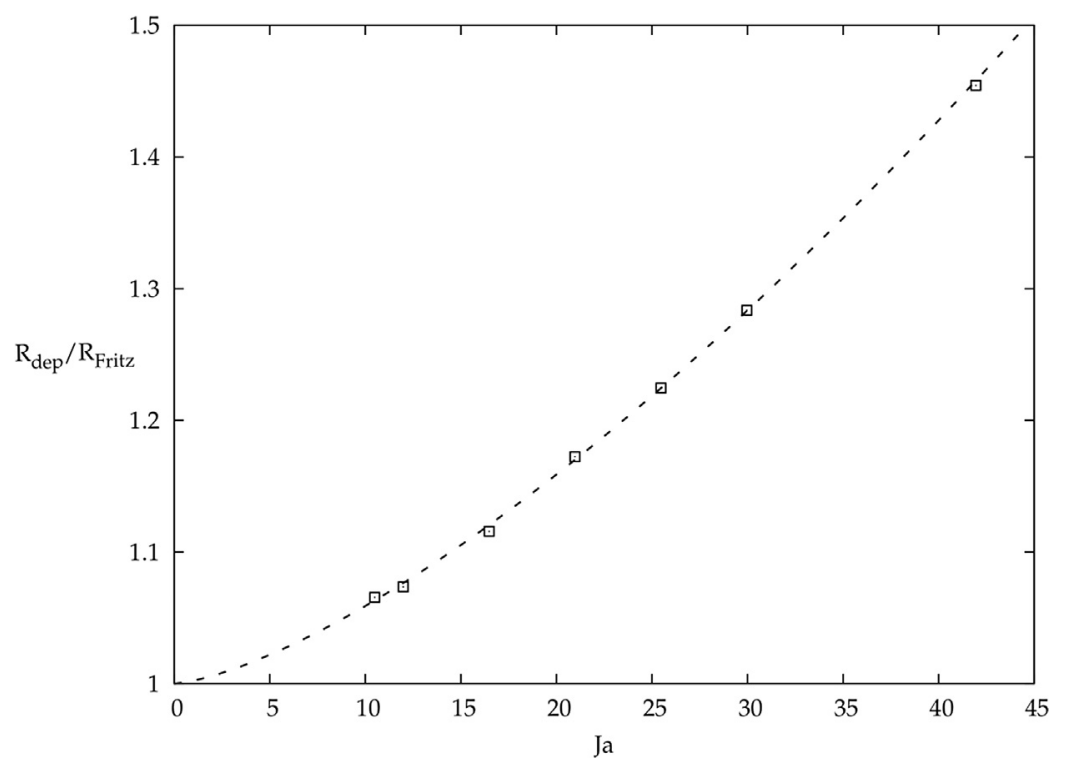

Fig. 23. Variation of the dimensionless departure radius with the Jakob number.

This expression implies that the bubble departure radius will be superior to the maximum static equilibrium radius. Indeed, after the static equilibrium radius has been reached, the bubble is still growing during the delay between the static equilibrium breakdown and the bubble departure. $\gamma$ is a dimensionless number accounting for the increase of the bubble departure dimensionless radius which can be expressed as a function of the dimensionless numbers whose bubble growth depends on, as proposed here,

$\gamma=f\left(J a, \operatorname{Pr}, \theta_{\text {micro }}, \frac{\delta}{R_{\text {fritz }}}, \frac{\rho_{l}}{\rho_{v}} \ldots\right)$

It is noteworthy, that no general correlation on the bubble departure radius exists in the literature whereas many experimental studies have been carried out. For instance, in page 244 in the Ref. [2], Carey proposes an overview of existing correlations, but none of them definitely settles this issue. This lack can be explained, considering the important experimental issues that must be faced to perform exhaustive experimental studies involving so many dimensionless numbers. As a consequence, the Direct Numerical Simulation seems to be currently a credible substitute to succeed such an achievement, since all the parameters can be easily varied when preforming numerical simulations. However this challenging task is out of the scope of this paper, and we will just propose here a simplified correlation based on the variations of one dimensionless number, i.e. the Jakob number, in order to illustrate the potential benefits that could be expected by this approach. The Jakob number has been modified by varying the latent heat of vaporization or the wall superheat. By performing this parametrical study the results obtained are summarized in Table 3. The variation of the dimensionless bubble departure radius $\frac{R_{\text {dep }}}{R_{\text {Fritz }}}$ with the Jakob number is plotted in Fig. 23 in order to deduce a correlation between these two variables. For the following values $\alpha=0.00219$ and $n=1.43$, the power law

$\frac{R_{\text {dep }}}{R_{\text {Fritz }}}=1+\alpha J a^{n}$

is the best fit of this curve. This correlation on the bubble departure diameter can be used in the configuration considered in this paper, i.e. moderate Jakob number, high contact angle and high density ratio. However, it should be mentioned that it is not complete and future works will be dedicated to express the coefficient $\alpha$ as a function of the other dimensionless numbers involved in nucleate pool boiling, such as those proposed in Eq. (50).

\section{Conclusions}

In this paper we have presented direct numerical simulations of nucleate pool boiling. Our solver is based on a well-established numerical methods previously validated for boiling flows [12,58] in a superheated liquid. A detailed numerical strategy is described by addressing several fundamental points that must be considered in the framework of nucleate boiling where a contact line appears. In a first time, a micro-region model for partially wetting liquids has been developed in order to quantify the influence of the wall superheat on the apparent contact angle and the contact line heat flux. We report that this influence is weak in our configurations, 
where moderate Jakob number, high contact angle and high density ratio are considered. Next, the grid sensitivity of the solution is fully investigated by comparing solutions obtained with four successive grids in order to verify that the simulations converge despite the thermal singularity between the interface temperature and the wall temperature. In the considered configuration, it is shown that the spatial convergence is reached with a cell size of about $10 \mu \mathrm{m}$. The weak influence of the micro region on the bubble growth is next confirmed by presenting satisfactory agreement for comparisons between numerical simulations and experimental data without using any micro region model. Finally, by coupling our fluid solver with thermal conduction solver in the solid domain, we show that the spatial variations of the thermal field in the substrate are not significant either, from which we can deduce that a simple isothermal boundary condition can be used without affecting the numerical results. It is noteworthy that the latter conclusion holds for thick substrate with a high thermal conductivity (small Biot number), but it could not remain valid at all for thinner substrates or small thermal conductivity (large Biot number). After these preliminary validations, we propose a detailed study on the temporal evolution of the forces acting on the bubble all along its growth. From this analysis, we can conclude that the bubble remains in a quasi-static equilibrium shape until it reaches its maximum static bubble radius, i.e. Fritz radius. Next dynamical effect does not remain negligible during the departure phase in which the bubble growth continues. Finally, by improving our understanding of the bubble growth and the departure mechanisms, we propose a simplified correlation on the dimensionless bubble detachment variation with the Jakob number. Although the scope of validity of this correlation is restrained to configurations close of the one considered in this paper, this work opens many important perspectives on more complete parametric studies involving a broader area of investigations by varying the other significant dimensionless numbers which are implied in Nucleate Pool Boiling.

\section{Conflict of interest}

None.

\section{Acknowledgements}

The authors gratefully acknowledge funding by CNES (Centre National des Etudes Spatiales) and by CNRS (Centre National de la Recherche Scientifique) for PhD Grant Support and CNES for Post-doctoral grant support.

\section{Appendix A. Resolution of the micro-region model}

The set of Eqs. (41)-(45) is solved from $s=0$ (contact line) to $s=L$, by using a 4 th order Runge Kutta algorithm. The solution depends on the fluid properties, the wall temperature and the microscopic contact angle. As a boundary condition is required to start the computation at the contact line $(s=0)$, we impose that the $x$ and $y$ coordinates are 0 , the angle is equal to the microscopic contact angle and the integrated heat flux is zero. An issue to address for solving this set of equations is to determinate the initial pressure jump. According to Eq. (43), the initial pressure jump depends on the triple line temperature which is also unknown. We can obtain this temperature by computing the initial pressure jump using a shooting method and an iterative method by imposing the following boundary condition on $s=L$

$\left.\frac{\partial \theta}{\partial s}\right|_{s=L}=0$.

First, we have to determine the value of $\Delta p_{s=0}$ to insure that the following boundary condition, $\left.\frac{\partial \theta}{\partial s}\right|_{s=L}=0$, will be preserved. This is done by using the secant numerical method:

$\Delta p_{s=0}^{n+1}=\Delta p_{s=0}^{n}-\frac{\Delta p_{s=0}^{n}-\Delta p_{s=0}^{n-1}}{\left.\frac{\partial \theta}{\partial s}\right|_{s=L} ^{n}-\left.\frac{\partial \theta}{\partial s}\right|_{s=L} ^{n-1}}$.

Next, once the value of $\Delta p_{s=0}$ has been determined, we can deduce the triple line temperature using Eq. (43). The starting boundary condition on the contact line, i.e. when $s=0$, are the following, $\quad x_{s=0}=0, \quad y_{s=0}=0, \quad \theta_{s=0}=\theta_{\text {micro }}, \quad \Delta p_{s=0}=\Delta p_{\text {shooting_method }}$, $Q_{s=0}=0$. At the other boundary of the computational domain, the following boundary condition $\left.\frac{\partial \theta}{\partial s}\right|_{s=L}=0$ is imposed, and the final values of each variables at $s=L$ are $x_{s=L}=x_{L}, y_{s=L}=y_{L}$, $\theta_{s=L}=\theta_{a p p}, \Delta p_{s=L}=\Delta p_{L}, Q_{s=L}=Q_{L}$. In order to validate the implementation of this solver, we present a comparison between Mathieu's model [35,36] and the model presented by Stephan and Büsse in [54] for perfectly wetting fluids. Whereas Mathieu's model has been designed for partially wetting fluids, we can just consider that the microscopic contact angle will tend to zero $\left(\theta_{\text {micro }}=10^{-6}\right)$ to simulate a perfectly wetting fluid. In Fig. 24 , we present a comparison between the two models, both on the integrated heat flux and on the contact angle, for a wall superheat
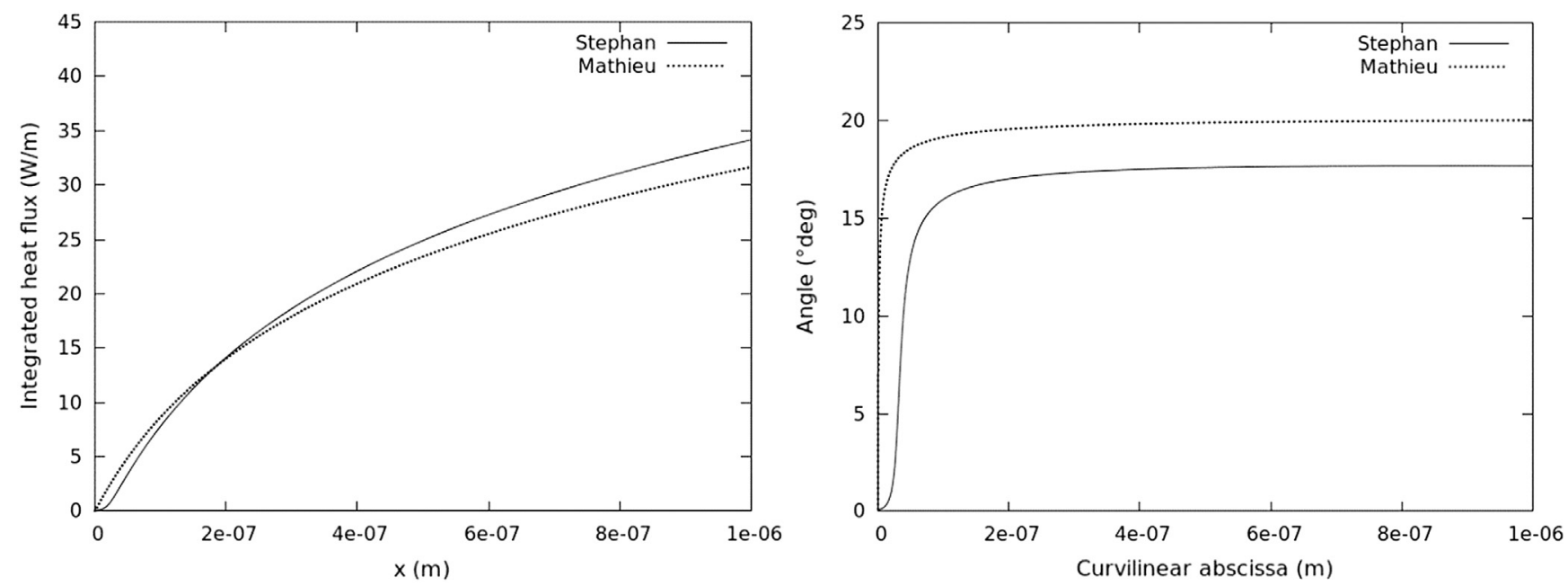

Fig. 24. Comparison between Mathieu model and Stephan and Busse model on the integrated heat flux and the contact angle for a zero microscopic contact angle 


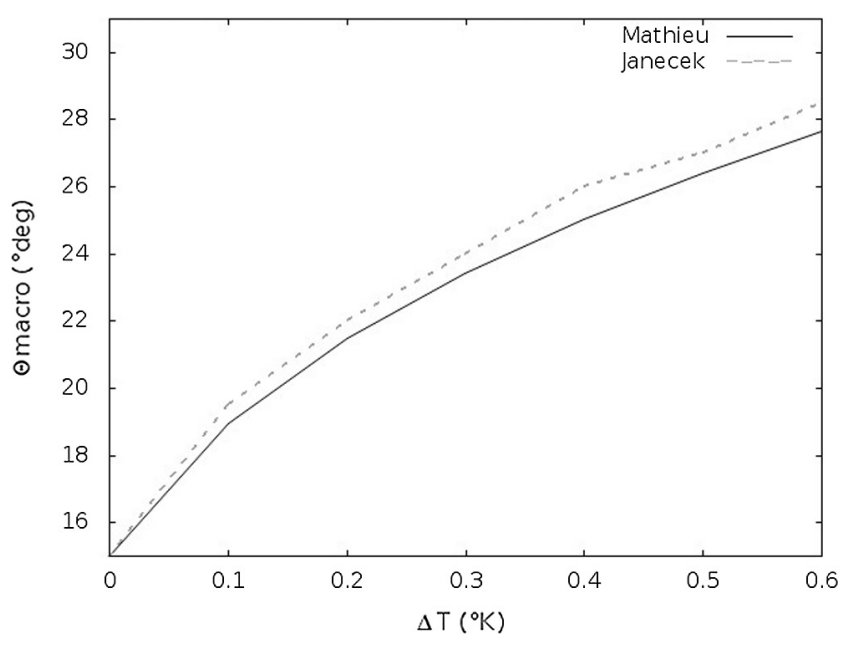

Fig. 25. Comparison between Mathieu model and Janececk model on the macroscopic contact angle for different wall superheat.

$\Delta T=7 \mathrm{~K}$. The two models exhibit similar behaviors for these two variables; especially a few differences exist on the value of the integrated heat flux. However if the contact angle is considered, a gap of about $4^{\circ}$ between the two models is observed.

A comparison between Mathieu's model and a micro region model presented by Janecek $[17,19]$ is also presented for a partially wetting fluid. As one conclusion of our study is about the weak influence of micro region effect in the configurations considered in the paper (contact angle up to $30^{\circ}$, moderate Jakob number, ambient pressure), we present now an assessment of Mathieu's model in a different configuration, still involving a partially wetting fluid, but with a significant influence of micro region effects on the contact angle. Such a behavior has been reported for high pressure applications by Janecek in a theoretical study [17]. A comparison between these two models is plotted in Fig. 25 for a simulation proposed in [17] involving high pressure (100 bars) water. In Fig. 25, we show the evolution of the apparent contact angle depending on the wall superheat. The agreement between the two models is good and brings a further validation to the global numerical methodology presented in the paper.

\section{Appendix B. Mechanical equilibrium of a static bubble contacting a wall}

Four forces are acting on a static bubble contacting an horizontal wall: the bubble weight, the liquid pressure force, the wall reaction to the vapor pressure and the capillary force [6,59]. The expressions of these different forces are detailed hereafter. The bubble weight can be expressed as

$$
\begin{aligned}
\vec{P} & =\rho_{v} V \vec{g}=\iiint_{V} \rho_{v} \vec{g} d V=-\iint_{S} p_{v} \vec{n} d S \\
& =-\iint_{S_{i}} p_{v} \vec{n} d S_{i}-\iint_{S_{w}} p_{v} \vec{n} d S_{w} .
\end{aligned}
$$

where $\mathrm{V}$ is the bubble volume, $S_{i}$ the surface of the interface, $S$ the closed surface around the vapor volume and $S_{w}$ is the dry surface on the wall. The other geometrical notations are described in Fig. 26. In

Eq. (55) the liquid pressure force $\vec{F}_{P}$ corresponds to the force of the liquid around the bubble acting on the interface

$$
\overrightarrow{\mathrm{F}}_{P}=\iint_{S_{i}} p_{l}(z) \vec{d} S_{I}
$$

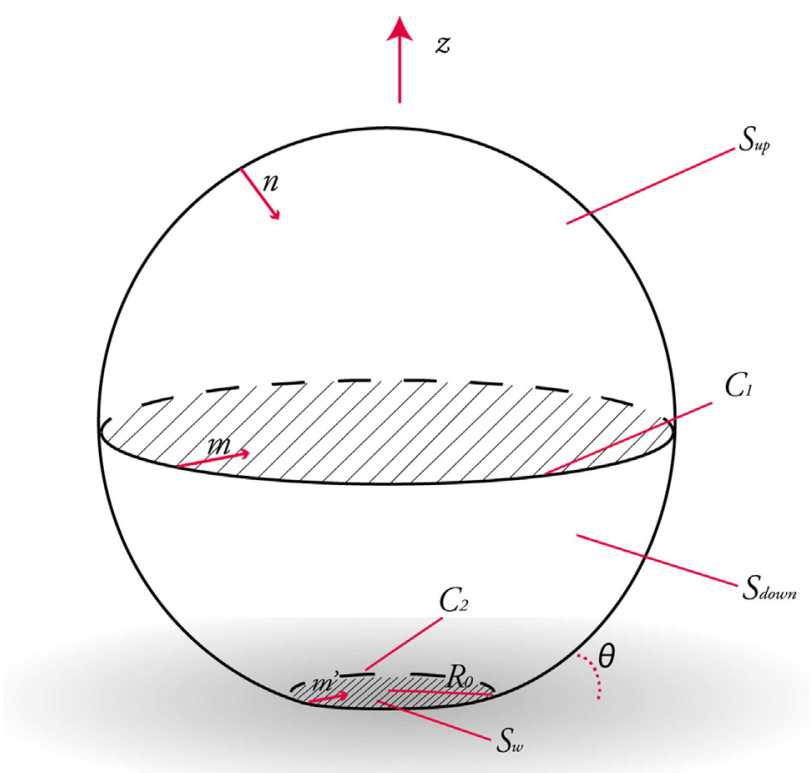

Fig. 26. Scheme of the bubble.

Assuming the hydrostatic equilibrium of the liquid surrounding the bubble, Eq. (55) reads:

$$
\overrightarrow{\mathrm{F}}_{P}=-\iiint_{V} \rho_{l} \vec{g} d V-\iint_{S_{w}} p_{l} \vec{n} d S_{w}=\vec{\Pi}-\pi R_{s}^{2} p_{l 0} \vec{e}_{z},
$$

where $\vec{\Pi}$ is the Archimede force:

$$
\vec{\Pi}=-\rho_{l} V_{b} \vec{g},
$$

where $p_{l 0}$ is the liquid pressure at the bubble foot. The wall reaction of the vapor pressure writes

$\vec{R}=\iint_{S_{w}} p_{v} \vec{n} d S_{w}=\pi R_{s}^{2} p_{v 0} \vec{e}_{z}$

And finally, the capillary force is

$\vec{C}=\iint_{S_{i}} \sigma \kappa(z) \vec{d} S_{i}=-2 \pi R_{0} \sin \theta \vec{e}_{z}$

The demonstration of Eq. (59) is detailed here. Because of the geometry of the problem, the total capillary force is directed along the z-axis. By integrating the z-projection of the capillary force

$\vec{C}=\iint_{S_{\text {up }}} \sigma \kappa(z) \vec{d} S_{i}+\iint_{S_{\text {down }}} \sigma \kappa(z) \vec{d} S_{i}$,

Using the Gauss theorem and the curvature definition $\kappa=\nabla \cdot \vec{n}$, the capillary force can be expressed as the sum of the two forces

$\iint_{S_{u p}} \sigma \kappa(z) \vec{d} S_{i}=\int_{C_{1}} \vec{n} \cdot \vec{m} d C_{1} \vec{e}_{z}$

$\iint_{S_{\text {down }}} \sigma \kappa(z) \vec{d} S_{i}=-\int_{C_{1}} \vec{n} \cdot \vec{m} d C_{1} \vec{e}_{z}+\int_{C_{2}} \vec{n} \cdot \vec{m}^{\prime} d C_{2} \vec{e}_{z}$,

which finally simplifies as

$\vec{C}=-2 \pi R_{0} \sin \theta \vec{e}_{z}$.

More details on this demonstration can be found in [41]. The static equilibrium condition is fulfilled if the sum of these forces is zero

$P^{\rightarrow}+\mathrm{F}_{P}^{\rightarrow}+R^{\rightarrow}+C^{\rightarrow}=0^{\rightarrow}$. 
The liquid pressure force and the vapor pressure reaction depends on the value of the pressure at the bubble foot. We can introduce also the contact pressure force which depends on the bubble foot pressure jump between the two phases

$$
\vec{F}_{c}=\pi R_{\mathrm{s}}^{2}\left(p_{v 0}-p_{l 0}\right) \vec{e}_{z}=\pi R_{\mathrm{s}}^{2} \sigma \kappa_{0} \vec{e}_{z},
$$

where $\kappa_{0}$ is the curvature of the interface at the bubble foot. The static equilibrium condition becomes

$$
P^{\rightarrow}+\Pi^{\rightarrow}+F_{c} \rightarrow+C^{\rightarrow}=0^{\rightarrow} .
$$

That can reformulated with the following surface integral of the Laplace law along the bubble interface

$\iint_{S_{i}}\left(p_{v}-p_{l}-\sigma \kappa\right) \vec{d} S_{I}=\overrightarrow{0}$

Although the expression of the liquid pressure force assumes the hydrostatic equilibrium, this pressure field can be disturbed by the bubble interface motion during the bubble growth. In order to quantify this phenomenon on the bubble force balance, we introduce the added mass force defined as the difference between the hydrostatic pressure field and the real pressure field:

$\vec{\omega}=\iint_{S_{i}}\left(p_{l, \text { real }}(z)-p_{l, \text { hydro }}(z)\right) \vec{d} S_{I}$

We show that the added masse force can be expressed as the opposite of the sum of the static forces

$\omega^{\rightarrow}=-\left(P^{\rightarrow}+\Pi^{\rightarrow}+F_{c} \rightarrow+C^{\rightarrow}\right)$.

The shape of a static bubble contacting a horizontal wall can be computed by using the local Laplace law. Due to the geometry of the problem, the bubble can be described in cylindrical coordinates. A numerical integration of this problem allows computing the shape and the maximum volume of a static bubble on a wall, as it has been previously shown by Fritz in [10]. It has been deduced from this numerical integration the following correlation on the maximum static bubble radius attached on a wall, known as the Fritz radius

$R_{F}=0.0104 \theta \sqrt{\frac{\sigma}{g\left(\rho_{L}-\rho_{V}\right)}}$.

\section{References}

[1] T. Aslam, A partial differential equation approach to multidimensiona extrapolation, J. Comput. Phys. 193 (2003) 349-355.

[2] V.P. Carey, Liquid-Vapor Phase Change Phenomena: An Introduction to the Thermophysics of Vaporization and Condensation Processes in Heat Transfe Equipment, Taylor \& Francis, 2007.

[3] C. Colin, O. Kannengieser, W. Bergez, M. Lebon, J. Sebilleau, M. Sagan, S. Tanguy, Nucleate Pool Boiling in microgravity: recent progress and future prospects, $\mathrm{C}$. R. Méc. 345 (1) (2017) 21-34.

[4] M. Cooper, Correlation for nucleate boiling-formulation using reduced pressure, Physicochem. Hydrodyn. 3 (1982) 89-111.

[5] A. Du Chéné, C. Min, F. Gibou, Second-order accurate computation of curvatures in a level set framework using novel high-order reinitialization schemes, J. Sci. Comput. 35 (2008) 114-131.

[6] G. Duhar, C. Colin, Dynamics of bubble growth and detachment in a viscous shear flow, Phys. Fluids 18 (2006) 077101.

[7] A. Esmaeeli, G. Tryggvason, A front tracking method for computations of boiling in complex geometries, Int. J. Multiphase Flow 30 (2004) 1037 1050.

[8] R. Fedkiw, T. Aslam, B. Merriman, S. Osher, A non-oscillatory Eulerian approach to interfaces in multimaterial flows (The Ghost Fluid Method), J. Comput. Phys. 152 (1999) 457-492.

[9] S. Fischer, T. Gambaryan-Roisman, P. Stephan, On the development of a thin evaporating liquid film at a receding liquid/vapour-interface, Int. J. Heat Mass Transfer 88 (2015) 346-356.

[10] W. Fritz, Maximum volume of vapor bubbles, Phys. Z. 36 (11) (1935) 379-384.

[11] F. Gibou, R. Fedkiw, L.T. Chieng, M. Kang, A second-order-accurate symmetric discretization of the Poisson equation on irregular domains, J. Comput. Phys. 176 (2002) 205-227.
[12] F. Gibou, L. Chen, D. Nguyen, S. Banerjee, A level set based sharp interface method for the multiphase incompressible Navier-Stokes equations with phase change, J. Comput. Phys. 222 (2007) 536-555.

[13] F. Gibou, C. Min, R. Fedkiw, High resolution sharp computational methods for elliptic and parabolic problems in complex geometries, J. Sci. Comput. 54 (2013) 369-413.

[14] S. Hänsch, S. Walker, The hydrodynamics of microlayer formation beneath vapour bubbles, Int. J. Heat Mass Transfer 102 (2016) 1282-1292.

[15] G. Huber, S. Tanguy, J.-C. Béra, B. Gilles, A time splitting projection scheme for compressible two-phase flows. Application to the interaction of bubbles with ultrasound waves. J. Comput. Phys. 302 (2015) 439-468.

[16] M. Ishii, T. Hibiki, Thermo-Fluid dynamics of two-phase flows, Springer, 2011.

[17] V. Janecek, Evaporation at Microscopic Scale and at High Heat Flux (PhD thesis), Université Pierre et Marie Curie, 2012.

[18] V. Janecek, B. Andreotti, D. Prazak, T. Barta, V.S. Nikolayev, Moving contact line of a volatile fluid, Phys. Rev. E 88 (2013) 060404(R).

[19] V. Janecek, V.S. Nikolayev, Apparent contact angle model at partial wetting and evaporation: impact of surface forces, Phys. Rev. E (87) (2013) 012404.

[20] G.S. Jiang, C.W. Shu, Efficient implementation of weighted essentially nonoscillatory schemes, J. Comput. Phys. 126 (1996) 202-228.

[21] D. Juric, G. Tryggvason, Computations of boiling flows, Int. J. Multiphase Flow 24 (3) (1998) 387-410

[22] M. Kang, R. Fedkiw, X.-D. Liu, A boundary condition capturing method for multiphase incompressible flow, J. Sci. Comput. 15 (2000) 323-360.

[23] W. Kays, M. Crawford, B. Weigand Convective Heat and Mass Transfer, fourth ed., Mc Graw Hill, 2004.

[24] J. Kim, Review of nucleate pool boiling bubble heat transfer mechanisms, Int. J. Multiphase Flow 35 (2009) 1067-1076.

[25] H. Kim, J. Buongiorno, Detection of liquid-vapor-solid triple contact line in two-phase heat transfer phenomena using high-speed infrared thermometry, Int. J. Multiphase Flow 37 (2011) 166-172.

[26] C. Kunkelmann, P. Stephan, Numerical simulation of the transient heat transfer during nucleate boiling of refrigerant HFE-7100, Int. J. Fluid Refrig. 33 (2010) 1221-1228.

[27] C. Kunkelmann, K. Ibrahen, N. Schweizer, S. Herbert, P. Stephan, T. GambaryanRoisman, The effect of three-phase contact line speed on local evaporative heat transfer: experimental and numerical investigations, Int. J. Heat Mass Transfers 55 (2012) 1896-1904.

[28] B. Lalanne, S. Tanguy, F. Risso, Effect of rising motion on the damped shape oscillations of drops and bubbles, Phys. Fluids 25 (2013) 112107.

[29] B. Lalanne, S. Tanguy, L. Rueda-Villegas, F. Risso, On the computation of viscous terms for incompressible flows with Level Set/Ghost Fluid Method, J. Comput. Phys. 301 (2015) 289-307.

[30] B. Lalanne, N.A. Chebel, J. Vejražka, S. Tanguy, O. Masbernat, F. Risso, Nonlinear shape oscillations of rising drops and bubbles: Experiments and simulations, Phys. Fluids 27(12) (2015) 123305.

[31] L.D. Landau, V. Levich, Dragging of a liquid by a moving plate, Acta Physiochim. USSR 17 (1942) 42-54.

[32] J. Lee, G. Son, A Level-set method for analysis of particle motion in an evaporating microdroplet, Numer. Heat Transfer, Part B 67 (2015) 25-46.

[33] M. Lepilliez, E.-R. Popescu, F. Gibou, S. Tanguy, On two-phase flow solver in irregular domains with contact line, J. Comput. Phys. 321 (2016) 12171251.

[34] X.-D. Liu, R. Fedkiw, M. Kang, A boundary condition capturing method for Poisson's equation on irregular domains, J. Comput. Phys. 160 (2000) 151-178.

[35] B. Mathieu. Études physique, expérimentale et numérique des mécanismes de base intervenant dans les écoulements diphasiques en micro-fluidique ( $\mathrm{PhD}$ thesis), Université de Provence, 2003.

[36] B. Mathieu, O. Lebaigue, L. Tadrist. Influence of a dynamic contact line model on the characteristics of nucleate wall boiling computed with a DNS approach, in: 5th International Conference of Multiphase Flow (ICMF 2004), Yokohama, Japan.

[37] C. Min, F. Gibou, A second order accurate level set method on non-graded adaptive Cartesian grids, J. Comput. Phys. 219 (2) (2006) 912-929.

[38] D. Nguyen, R. Fedkiw, M. Kang, A boundary condition capturing method for incompressible flame discontinuities, J. Comput. Phys. 172 (2001) 71-98.

[39] Y.T. Ng, C. Min, F. Gibou, An efficient fluid-solid coupling algorithm for singlephase flows, J. Comput. Phys. 228 (23) (2009) 8807-8829.

[40] V. Nikolayev, Dynamics of the triple contact line on a non-isothermal heater at partial wetting, Phys. Fluids 22 (2010) 082105.

[41] V. Nikolayev, V. Janeček, Impact of the apparent contact angle on the bubble departure at boiling. Int. J. Heat Mass Transfer 55 (2012) 7352-7354.

[42] S. Osher, J.A. Sethian, Fronts propagating with curvature-dependent speed: algorithms based on Hamilton-Jacobi formulations, J. Comput. Phys. 79 (1988) $12-49$.

[43] J. Papac, F. Gibou, C. Ratsch, Efficient symmetric discretization for the Poisson, heat and Stefan-type problems with Robin boundary conditions, J. Comput. Phys. 229 (2010) 875-889.

[44] A. Rednikov, P. Colinet, Singularity-free description of moving contact lines for volatile liquids, Phys. Rev. E (2013) 010401(R).

[45] L. Rueda Villegas, R. Alis, M. Lepilliez, S. Tanguy, A. Ghost Fluid-Level Set method for boiling flows and liquid evaporation. Application to the Leidenfrost effect. J. Compit. Phys. 316 (2013) 789-813.

[46] L. Rueda Villegas, S. Tanguy, G. Castanet, O. Caballina, F Lemoine. Direct numerical simulation of the impact of a droplet onto a hot surface above the Leidenfrost temperature, Int. J. Heat Mass Transfer 104 (2017) 1090-1109. 
[47] G. Russo, P. Smereka, A remark on computing distance functions, J. Comput. Phys. 163 (1) (2000) 51-67.

[48] Y. Sato, B. Niceno, A sharp-interface phase change model for a massconservative interface tracking method, J. Comput. Phys. 249 (2013) 127-161.

[49] Y. Sato, B. Niceno, A depletable micro-layer model for nucleate pool boiling, J. Comput. Phys. 300 (2015) 20-52.

[50] R. Scardovelli, S. Zaleski, Direct numerical simulation of free-surface and interfacial flow, Annu. Rev. Fluid Mech. 31 (1999) 567-603.

[51] G. Son, V.K. Dhir, Numerical simulation of film boiling near critical pressures with a level set method, J. Heat Transfer 120 (1998).

[52] G. Son, V.K. Dhir, N. Ramanujapu, Dynamics and heat transfer associated with a single bubble during nucleate boiling on a horizontal surface, J. Heat Transfer 121 (1999) 623-631.

[53] K. Stephan, M. Abdelsalam, Heat-transfer correlation for natural convection boiling, Int. J. Heat Mass Transfer 23 (1980) 73-87.

[54] P.C. Stephan, C.A. Busse, Analysis of the heat transfer coefficient on grooved heat pipe evaporator walls, Int. J. Heat Mass Transfer 35 (1992) 383-391.
[55] M. Sussman, P. Smereka, S. Osher, A level set approach for computing solutions to incompressible two-phase flow, J. Comput. Phys. 114 (1994) 146-159.

[56] M. Sussman, K.M. Smith, M.Y. Hussaini, M. Ohta, R. Zhi-Wei, A sharp interface method for incompressible two-phase flows, J. Comput. Phys. 221 (2007) 469505.

[57] S. Tanguy, T. Menard, A. Berlemont, A level set method for vaporizing twophase flows, J. Comput. Phys. 221 (2007) 837-853.

[58] S. Tanguy, M. Sagan, B. Lalanne, F. Couderc, C. Colin, Benchmarks and numerical methods for the simulation of boiling flows, J. Comput. Phys. 264 (2014) 1-22.

[59] G.E. Thorncroft, J.F. Klausner, R. Mei, Bubble forces and detachment models, Multiphase Sci. Technol. 13 (2001) 35-76.

[60] P.C. Wayner, Y.K. Kao, L.V. LaCroix, The interline heat-transfer coefficient of an evaporating wetting liquid film, Int. J. Heat Mass Transfer 19 (1976) 487-492.

[61] S. Welch, J. Wilson, A volume of fluid based method for fluid flows with phase change, J. Comput. Phys. 160 (2000) 662-682. 